Document downloaded from:

http://hdl.handle.net/10251/122535

This paper must be cited as:

Peña-Monferrer, C.; Monrós Andreu, G.; Chiva Vicent, S.; Martinez-Cuenca, R.; MuñozCobo, JL. (2018). A CFD-DEM solver to model bubbly flow. Part I: Model development and assessment in upward vertical pipes. Chemical Engineering Science. 176:524-545. https://doi.org/10.1016/j.ces.2017.11.005

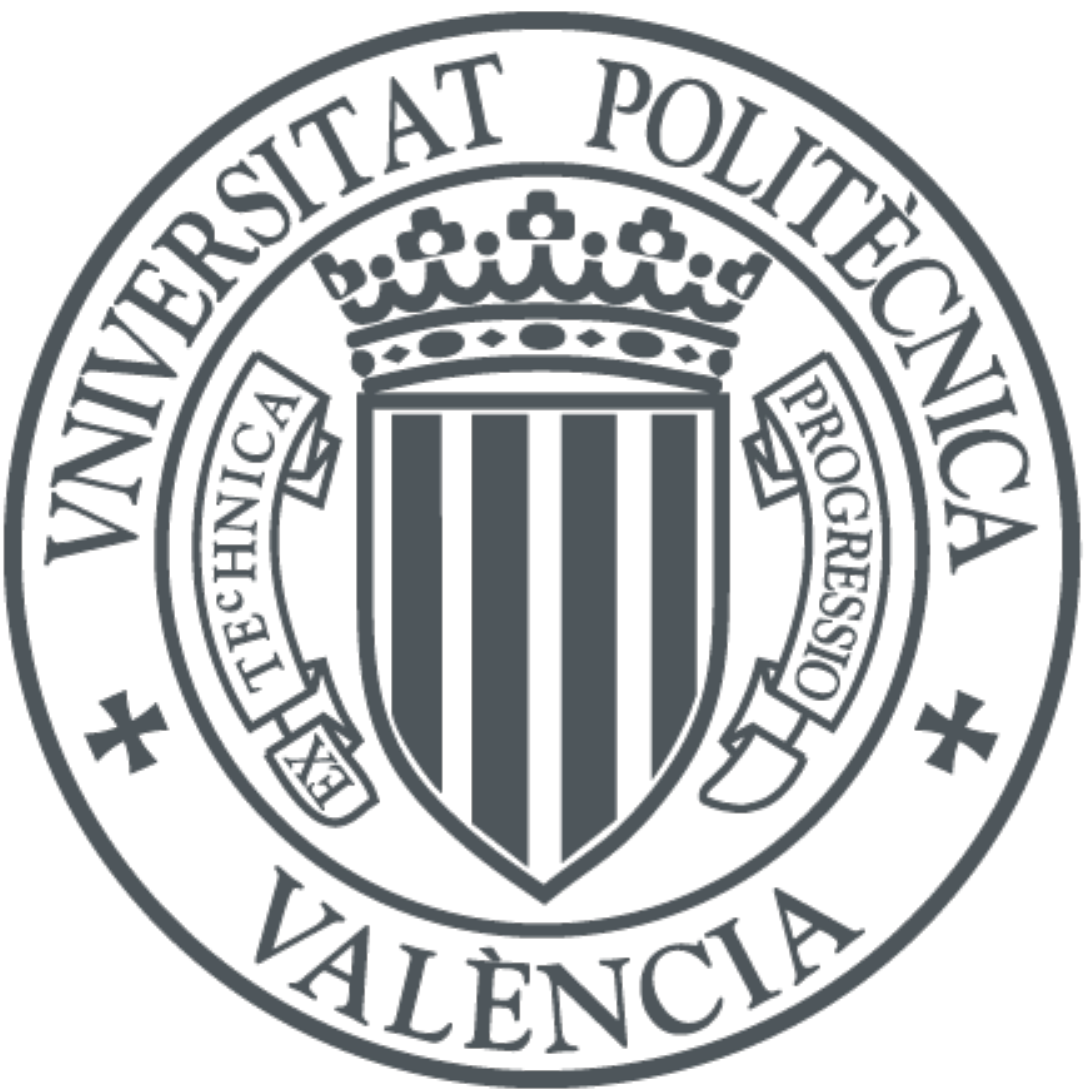

The final publication is available at

https://doi.org/10.1016/j.ces.2017.11.005

Copyright Elsevier

Additional Information 


\section{Accepted Manuscript}

A CFD-DEM solver to model bubbly flow. Part I: Model development and assessment in upward vertical pipes

C. Peña-Monferrer, G. Monrós-Andreu, S. Chiva, R. Martínez-Cuenca, J.L. Muñoz-Cobo

PII: S0009-2509(17)30684-X

DOI: https://doi.org/10.1016/j.ces.2017.11.005

Reference: CES 13891

To appear in: Chemical Engineering Science

Received Date: 16 May 2017

Revised Date: 15 September 2017

Accepted Date: 3 November 2017

Please cite this article as: C. Peña-Monferrer, G. Monrós-Andreu, S. Chiva, R. Martínez-Cuenca, J.L. Muñoz-Cobo, A CFD-DEM solver to model bubbly flow. Part I: Model development and assessment in upward vertical pipes, Chemical Engineering Science (2017), doi: https://doi.org/10.1016/j.ces.2017.11.005

This is a PDF file of an unedited manuscript that has been accepted for publication. As a service to our customers we are providing this early version of the manuscript. The manuscript will undergo copyediting, typesetting, and review of the resulting proof before it is published in its final form. Please note that during the production process errors may be discovered which could affect the content, and all legal disclaimers that apply to the journal pertain. 


\title{
A CFD-DEM solver to model bubbly flow. Part I: Model development and assessment in upward vertical pipes
}

\author{
C. Peña-Monferrer ${ }^{\mathrm{a}, \mathrm{b}, *}$, G. Monrós-Andreu ${ }^{\mathrm{a}}$, S. Chiva ${ }^{\mathrm{a}}$, R. Martínez-Cuenca ${ }^{\mathrm{a}}$, J.L. Muñoz-Cobo ${ }^{\mathrm{b}}$ \\ ${ }^{a}$ Department of Mechanical Engineering and Construction, Universitat Jaume I. Campus del Riu Sec, 12080 Castelló de la Plana, Spain \\ ${ }^{b}$ Institute for Energy Engineering, Universitat Politècnica de València. Camí de Vera, s/n, 46022 València, Spain
}

\begin{abstract}
In the computational modeling of two-phase flow, many uncertainties are usually faced in simulations and validations with experiments. This has traditionally made it difficult to provide a general method to predict the two-phase flow characteristics for any geometry and condition, even for bubbly flow regimes. Thus, we focus our research on studying in depth the bubbly flow modeling and validation from a critical point of view. The conditions are intentionally limited to scenarios where coalescence and breakup can be neglected, to concentrate on the study of bubble dynamics and its interaction with the main fluid. This study required the development of a solver for bubbly flow with higher resolution level than TFM and a new methodology to obtain the data from the simulation. Part I shows the development of a solver based on the CFD-DEM formulation. The motion of each bubble is computed individually with this solver and aspects as inhomogeneity, nonlinearity of the interfacial forces, bubble-wall interactions and turbulence effects in interfacial forces are taken into account. To develop the solver, several features that are not usually required for traditional CFD-DEM simulations but are relevant for bubbly flow in pipes have been included. Models for the assignment of void fraction into the grid, seeding of bubbles at the inlet, pressure change influence on the bubble size and turbulence effects on both phases have been assessed and compared with experiments for an upward vertical pipe scenario. Finally, the bubble path for bubbles of different size have been investigated and the interfacial forces analyzed.
\end{abstract}

Keywords: CFD-DEM, OpenFOAM ${ }^{\circledR}$, two-phase flow, bubbly flow, soft-sphere model, continuous random walk model

\section{Introduction}

Gas-liquid two-phase flow is present in natural and industrial processes of different nature as chemical and nuclear reactors, oil flow or heat exchangers. Because of the importance predicting the flow structure using computational methods, two-phase flow has been investigated over the years becoming a research focus with important strides carried out to date.

Among the multiple flow configurations or regimes, the bubbly flow is the simplest one. Typically, spherical or ellipsoidal bubbles compose the disperse phase moving across the main continuous phase. Bubbly flow is one of the most common flow regime, existing in industrial systems as it is the case of bubble column reactors, aerators or pipeline transport. In vertical pipes, flow maps are traditionally used to predict the patterns as a function of superficial velocities or superficial momentum flux (Taitel et al., 1980). Bubbly flow is given at low gas-liquid superficial velocities according to these maps. However, depending on the bubble size at the injection, or the carrier phase solution, the pattern boundaries could change. A more general definition of flow regime is given by Besagni

\footnotetext{
* Corresponding author

Email addresses: cpena@uji.es (C. Peña-Monferrer), gmonros@emc.uji.es (G. Monrós-Andreu), schiva@emc.uji.es (S Chiva), rcuenca@emc.uji.es (R. Martínez-Cuenca), jlcobos@iqn.upv.es (J.L. Muñoz-Cobo)
}

et al. (2017); Guédon et al. (2017). Four flow patterns were differentiated for vertical pipes: homogeneous, heterogeneous, slug or annular flow regime. The bubbly flow term used in the present work meets the homogeneous flow regime definition where noncoalescence-induced bubbles are present.

Despite its apparent simplicity, the challenges for modeling bubbly flow in simple geometries are various. The problems for modeling arise mainly from the difficulty to obtain universal correlations for the interfacial forces and to predict the turbulence effect produced by the bubbles. The breakup and coalescence phenomena further complicate the simulation as they strongly influence bubble diameters and void evolution. Also, for wall-bounded flow systems, the wall largely influence the disperse phase, determining the characteristics of the two-phase flow in the whole domain. This influence becomes important in pipes with small diameter compared with large diameter pipes or bubble columns. The turbulence inhomogeneity, the twophase flow boundary layer effects and the interaction of the bubbles with the wall are relevant in these systems.

All these challenges have a strong impact on the simulation literature. On one hand, there is no general agreement on which correlations should be used, and every work uses a specific set of models and coefficients. On the other hand, measurements of two-phase flow variables is also a complex task that needs to be handled from a very critical point of view. In other words, the coefficients in the simulations are tuned so the results match as close as possible the data sets with no consideration on the 
data reliability.

Computational simulations employing different approaches have been used in the past to predict the two-phase flow behavior at different resolution levels. Thus, the literature contains various developments at microscopic, mesoscopic and macroscopic levels as Interface capturing or tracking Methods (IM), Discrete Element Method (DEM) or Two-Fluid Method (TFM) respectively (Ishii and Hibiki, 2006; Prosperetti and Tryggvason, 2007).

These different approaches can be employed to apply different levels of modeling, to study phenomena at a certain length scale (Deen et al., 2004; Gunsing, 2004). Higher resolution level approaches provide generally more confident results. Then, they can be suitable for complex scenarios when computational requirements allow their use. In addition, the same scenario can be simulated with different approaches, then a multiscale study may reveal useful outcomes to investigate interfacial closures. In vertical pipes, little research has been done with a higher resolution level approach than TFM. An exhaustive study of bubbly flow in vertical pipes with CFD-DEM is of interest for both the simulation of complex problems and the multiscale modeling.

\subsection{Bubbly flow and computational methods}

Simulations involving gas-liquid two-phase flow systems have been performed in the past trying to reproduce the experimental results. Most detailed simulation approaches, as IM, may resolve the individual bubble interfaces and complex phenomena as their deformation by fluid forces. To apply these approaches to industrial scale systems, is nowadays hardly possible as they require excessive computational time and memory resources. Thousands or even millions of bubbles may be present in these systems.

For these applications, TFM is often preferred given its reduced computational cost. Several efforts have been done in the past to simulate bubble columns (Gupta and Roy, 2013; Pan et al., 1999; Pfleger et al., 1999; Zhang et al., 2006) or pipes (Hosokawa and Tomiyama, 2009; Krepper et al., 2005; Ekambara et al.,2008; Peña-Monferrer et al., 2016b) using the TFM.

From these efforts, it has become apparent the drawbacks of these simulations. They are dependent on the set of correlations used for the interfacial force models and coefficients, breakup and coalescence and turbulence modeling. These sets vary widely among the different works. The main reasons for these differences are related to the difficulty of modeling twophase flow phenomena with phase average equations. The averaging of the disperse phase results in loss of valuable information needed to describe complex physical phenomena such as bubble swarms interactions and bubble collisions. Then, these complex phenomena need to be modeled by using correlations that have been proven to work in a reduced set of flow conditions.
Even in simple geometries and low void fractions, a complex flow behavior, that implies difficulties for the modeling, is observed. For instance, we show the Video S1 and Video S2. The images are captured with a high speed camera in a vertical pipe of diameter $52 \mathrm{~mm}$ at $j_{\mathrm{c}}=0.5 \mathrm{~m} / \mathrm{s}$ and $j_{\mathrm{d}}=0.03 \mathrm{~m} / \mathrm{s}$. In these videos we can note, at a first glance, different rising velocities of the bubbles. The turbulence effects are noted in the bubble motion producing a lateral movement. Close to the wall bubble bounces are appreciated. As an example, Video S2 shows the rise of some bubbles interacting with the wall. In the images is appreciated how the second bubble pointed out with an arrow collides with the wall. In fact, it is shown how the bubble's side in contact with the wall is detached and a small oscillation in the bubble's surface is produced. After that, the bubble moves far from the wall. This effect is mentioned by Alajbegovic et al. (1999), noting that the bubble-wall force would be responsible of moving the bubbles to the flow stream. This behavior is also noted by de Vries (2001). The latter studied the bubble-wall interactions demonstrating how a bubble can bounce repeatedly against the wall, or departs away from the wall, depending on the bubble size. These observations indicate that higher resolution level approaches could be useful to investigate the modeling of these scenarios.

At the mesoscopic level, the Lagrangian-Eulerian (LE) formulation is a middle option between IM and TFM. It needs less computational cost than IM but fewer assumptions than TFM. The classical LE methods do not resolve the bubble interfaces, but many bubbles can be computed. LE can capture complex physical phenomena such as the nonlinear, multiscale interactions and nonequilibrium effects in multiphase flow (Fox, 2012; Subramaniam, 2013). The bubble paths are computed and complex phenomena as particle-particle interactions, particle-wall interactions, coalescence or breakup can be better described than with macroscopic models. Using a method relying on a discrete model has the advantage to be able to consider the following aspects inherently in the method:

a) Inhomogeneity of the disperse phase flow as the motion of each bubble is computed individually. This implies that the nonlinearity of the bubble forces can be accounted for. This is especially important for the lift force, but also for drag or bubble-wall contact forces.

b) Interfacial force coefficients that were obtained experimentally for individual bubbles are applied directly in the simulation.

c) Bubble-bubble and bubble-wall interactions can be computed in a mechanistic way. This allows the computation of elastic collisions and avoids the need for modeling parameters as the collision frequency.

d) Turbulent effects on all the bubble forces can be considered directly. 
Note that the mentioned above is usually not considered with TFM as the modelling becomes more complex or unfeasible as it is not clear nowadays that the required relationships can be found. For instance, a simulation of bubbly flow trough a perforated plate with TFM and CFD-DEM approaches was performed in Peña-Monferrer et al. (2016a) revealing important discrepancies between both methods.

The LE approach started some decades ago as a tool for the numerical simulation of sprays (Dukowicz, 1980; O'Rourke, 1981, 1985). O'Rourke developed a new approach coupling the Lagrangian equation of Williams (1958) for the disperse phase with an Eulerian description for the continuous phase. In their calculations velocity and pressure were obtained by means of the Navier-Stokes equations. The motion of each particle was solved using the Newton's second law including the particle-particle and particle-wall interactions, coalescence and breakup. In addition, a two-way coupling model for particlefluid was developed by Dukowicz to consider the effect of the disperse phase into the continuous phase. This method has been mainly applied to sprays or particle-laden flows. Further and in a lesser extent LE were applied to bubbly flows applications (Delnoij et al., 1997; E. Shams et al., 2010; Essa, 2012). The reader is referred to Subramaniam (2013) for more in-depth details about LE methods.

Within the LE formulation holds the CFD-DEM method usually characterized to consider the elastic collisions of the particles based on a soft-sphere model. CFD-DEM model has traditionally been applied to dense flow systems (Matuttis et al., 2000; Alam and Luding, 2003). However, could result especially useful to wall-bounded systems where the particle interactions play a crucial role as in the two-phase pipe flow investigated in this work.

The CFD-DEM approach has been extensively used to simulate two-phase flow systems as particle-laden flows, but new improvements to develop a new solver are needed to simulate bubbly flows accurately. In bubbly flow there are key differences compared to particle-laden flows that need to be accounted. Magnaudet and Eames (2000) noted three important differences that are listed below in words of the author:

a) "When the liquid is pure enough, it has the possibility to slip along the surface of the bubbles, in contrast to the flow past rigid bodies where the nonslip conditions is imposed."

b) "Owing to the very weak relative density of bubbles compared to that of the liquid, almost all the inertia is contained in the liquid, making inertia-induced hydrodynamic forces particularly important in the prediction of bubble motion."

c) "The shape of the bubbles can change with the local hydrodynamics"

The foregoing has several consequences in the requirements of the solver. First, in the computation of the interfacial forces, 255 the modeling is more complicated in order to represent the real 256 behavior of the bubbles. Second, the coupling between both 257
${ }_{14}$ phases including turbulence effects of the bubbles into the liquid and vice versa will have a relevant influence into the flow characteristics. Finally, the measurements of the bubble variables are in general more complicated.

\subsection{Objectives and outline}

The objective of the present work is to develop a confident solver to predict vertical bubbly flow. A general aim is that the results were accurate for pipes of different diameters and flow conditions without needing tuning coefficients. The solver relies on the CFD-DEM approach for unresolved particles. This implied that improvements were needed to simulate bubbly flows accurately. In particular, the void fraction calculation on the grid, seeding algorithm, bubble expansion and turbulence effects on both liquid and bubbles. Because of the high importance and complexity of modeling accurately the two-phase flow, we intentionally limited this work to scenarios where the breakup and coalescence effects can be neglected. For instance, from the experimental data analyzed by the camera, no evidence of coalescence or breakup was found for this condition. This allows investigating in depth the bubble dynamics, and results suitable to interpret more accurately the results and validations.

The paper is organized as follows. Section 2 describes the experimental facility and measurement techniques. A new experiment was performed to obtain complementary data using the experimental facility described in Monrós-Andreu et al. (2013, 2017). The main mathematical formulation and methods are described in Section 3. To develop the solver, a CFD-DEM approach for unresolved particles was used to extend its use to bubbly flow. The solver has been developed and implemented in the framework of the open source package OpenFOAM ${ }^{\circledR}$. Its libraries and methods served as a basis to develop this solver. Section 4 is focused on the methods to assign the volume fraction and momentum in the mesh used to solve the liquid phase. An equivolumetric subelement method to improve precision and accuracy is presented. Section 5 deals with the algorithm to seed the bubbles in a circular pipe for a given polydisperse flow. This algorithm allows reproducing experimental conditions adequately as air flow rate, BSD, bubble velocity, bubble frequency and void fraction profile. In Section 6, we analyze the effects of the bubble size distributions on the flow behavior. First, the assumption of monodispersity and polydispersity is analyzed to study its influence on the computational results. Also, the bubble expansion because of the pressure change as a function of the height was examined, measuring experimentally the bubble size at different measurement ports. The bubbles generate random velocity fluctuations in the flow that were modeled in this work using a two-phase flow $k-\varepsilon$ turbulence model. At the same time, the turbulence has an effect on the bubbles motion that was described with a Continuous Random Walk (CRW) model. This was studied in conjunction in Section 7. Section 8 analyzes the effect of turbulence and bubble size on the bubble's path and the influence of the lateral forces. Finally, Section 9 summarizes the conclusions of this study. The Appendix presents the mesh sensitivity analysis for this study. 


\section{Description of the experimental data and measurement techniques}

To extend the possibilities of validation of the solver, we performed a new set of experiments to fulfill the following requirements:

a) The pipe has the necessary length to note the flow characteristics evolution even under low void fraction conditions.

b) The data has enough information to test the solver and the involved models with high spatial resolution at different axial locations. The following variables are shown: bubble velocity, void fraction, interfacial area concentration, chord length, Sauter mean diameter, bubble size distribution, bubble frequency, missing ration, liquid velocity and turbulence.

c) The probability density function of the variables is available.

d) The data contains error bars to account for accuracy and precision through repeated observations which is valuable to analyze the computational results.

This section describes the experimental facility and measurement techniques used. For further details about the measurement of liquid and gas phase variables, the reader is referred to Part II of this paper (Peña-Monferrer et al., 2017).

\subsection{Experimental facility}

The experimental data is based on the facility described in Monrós-Andreu et al. (2013, 2017). The experimental facility is located at the Laboratory of Hydraulics of the Universitat Jaume I. It consists of an upward flow experimental loop (Fig. 1) with three axial locations used for the measurements: $\mathrm{z} / \mathrm{D}=22.4, \mathrm{z} / \mathrm{D}=61.0$ and $\mathrm{z} / \mathrm{D}=98.7$.

The facility has a pipe of diameter $52 \mathrm{~mm}$, length $5500 \mathrm{~mm}$ and a sparger to inject the air flow. The sparger (Mott Corp., 850 Series) is made of porous $316 \mathrm{~L}$ stainless steel. Its elements have a $13 \mathrm{~mm}(1 / 2 \mathrm{inch})$ diameter and mean pore size of $10 \mu \mathrm{m}$.

A centrifugal pump was used to circulate Osmotized water (200-300 $\mu \mathrm{S} \mathrm{m}^{-1}$ ) stored in a $500 \mathrm{~L}$ reservoir tank. The tank is kept at a constant temperature thanks to a heat exchanger. The water flow rate introduced in the system was measured by an electromagnetic flowmeter (M1000, Badger Meter Inc). An air flowmeter controller (EL-FLOW 250 Nlpm, Bronckhorst HiTech) was used to adjust and measure the gas flow rate.

\subsection{Measurement techniques}

In addition to the flowmeters, measurement equipment is located at different locations along the pipe. It consists of conductivity probes, Laser Doppler Anemometry (LDA), pressure transducers and High-Speed Cameras (HSC). A scheme of the test rig is shown in Fig. 2.

Four-sensor conductivity probes and Laser Doppler Anemometry (LDA) techniques were adopted to extract ${ }_{32}$ information from the air-water flow field. The measurement 321 sosimultaneously recorded over 30 seconds at $60 \mathrm{kHz}$ individual

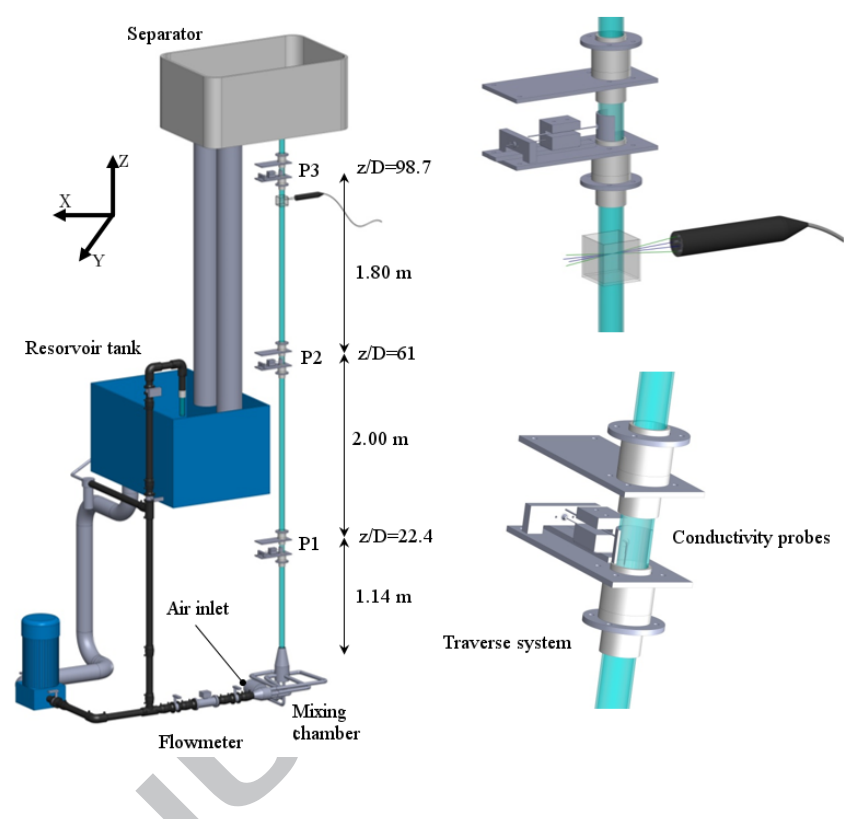

Figure 1: Experimental facility.

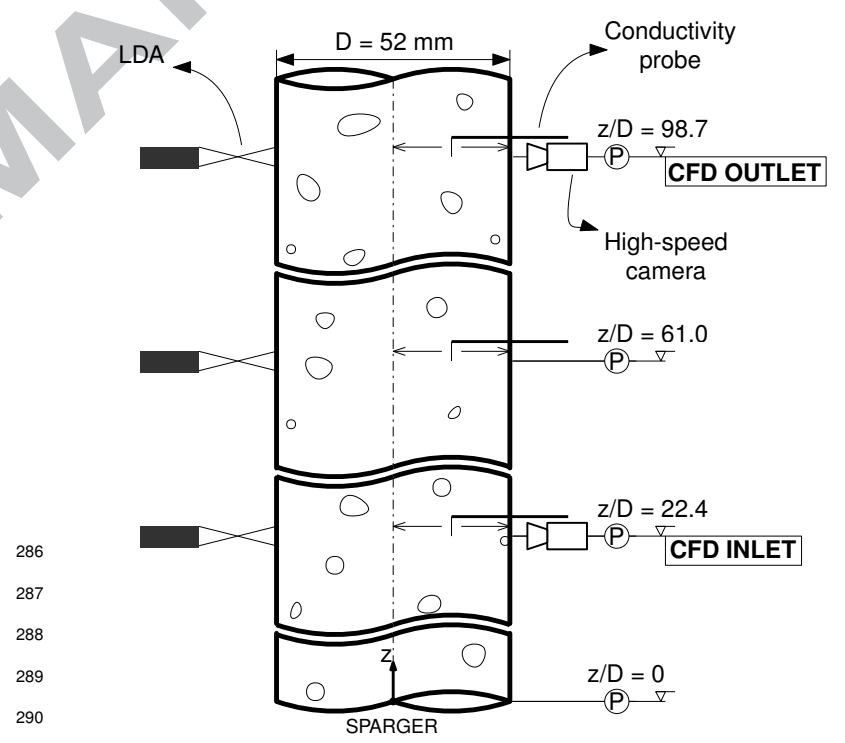

${ }^{291}$ Figure 2: Experimental configurations of the three two-phase flow systems used.

293 294

${ }^{295}$ system consisted of three mounted four-sensor conductivity ${ }^{29}$ probes, mechanical traverses, a measurement circuit, a digital ${ }^{29}$ high-speed acquisition board, and the software used for signal ${ }^{29} \mathrm{p}$ rocessing. The four-sensor conductivity probe was attached to the mechanical traverses mounted on a customized flange. ${ }^{29} \mathrm{It}$ can be moved along the radial direction of the test section sodusing controlled step motors. The measurement circuit was zoused to measure the voltage difference between the exposed 3otip and the grounded terminal. A high-speed acquisition board ${ }_{30}$ (National Instrument Corp., SCXI-1325) and a PC were used 3oto acquire the signals of the four-sensor probe, with a control 3oprogram developed under the LabView (National Instrument ${ }_{30}$ Corp.) software environment. Probe voltage signals were 
probe tip sampling rate $(720 \mathrm{kHz}$ total sampling rate considering the three sensors). Around 300 to 900 bubbles are detected by each probe at the different radial positions. To analyze the convergence of the average values obtained in the experiments, we monitored the time evolution of the average void fraction at different radial positions.

To measure the BSD, we located high-speed cameras at bottom $(\mathrm{z} / \mathrm{D}=22.4)$ and top $(\mathrm{z} / \mathrm{D}=98.7)$ ports. Frames of the images obtained are shown in Fig. 3.
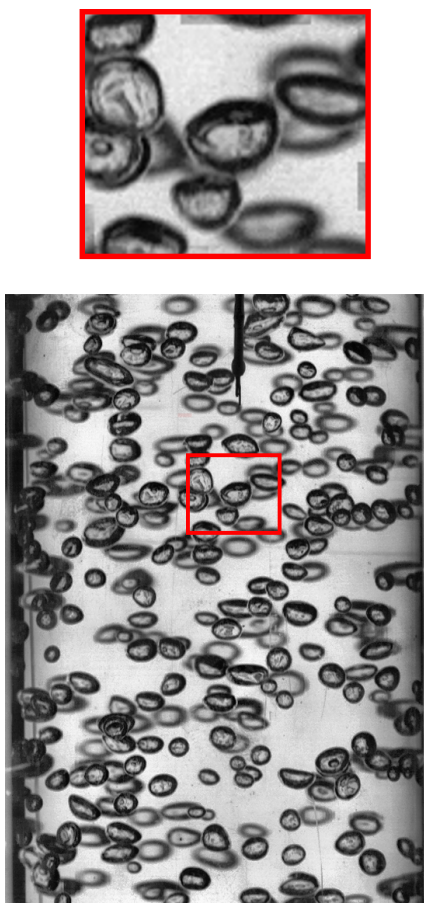
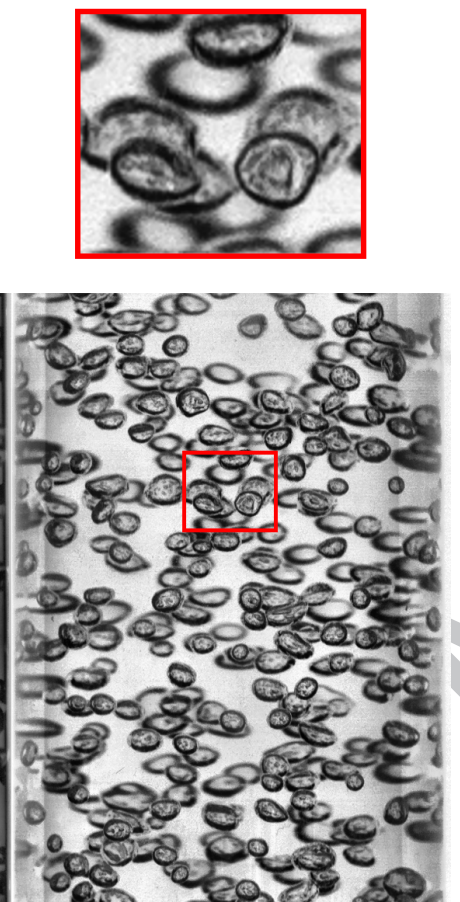

Figure 3: Image obtained by the high-speed camera for PW05003 at $\mathrm{z} / \mathrm{D}=22.4^{363}$ (left) and $\mathrm{z} / \mathrm{D}=98.7$ (right).

The images were taken by placing a IDT NX4-S2 high-speed camera approximately $1 \mathrm{~m}$ away from the test section axis. The sensor is a complementary metal-oxide semiconductor matrix composed by $1024 \times 1024$ pixels able to shot at $2000 \mathrm{fps}$ at full resolution $(2500 \mathrm{~Hz}$ were achieved by choosing a reduced matrix of $608 \times 784$ pixels). The AF-S Nikkor 18-70 mm 1:3.5-4.5 lens was used as imaging lens. The focal length was set to fit the entire width of the test section in the image and the aperture stop was fully opened (approximately f4). In this configuration a spatial resolution of approximately $9.17 \mathrm{px} / \mathrm{mm}$ was measured (the dimensions of the immersed conductivity probes served to compute the pixel size on the imaging plane). The backlight illumination was provided by a $5000 \mathrm{~lm}$ diffusing LED panel (60 x $60 \mathrm{~cm}$ ) and the exposure time was set to $398 \mu \mathrm{s}$. Note that, in order to minimize field distortions due to the cylindrical geometry of the pipe, a squared methacrylate box filled with water 365 was placed surrounding the test section at the imaging location. The axis of the camera was aligned orthogonal to one of the 366 planar surfaces of the box.

Nearly 500 bubbles were manually measured for every port 368 (Fig 4). Several points in the bubble borders were selected. An ellipse is fitted to the selected points by using a least-squares $s_{370}$ algorithm that provides with both axis and orientation angle. The semi-axis are used to obtain the equivalent diameter for each bubble.

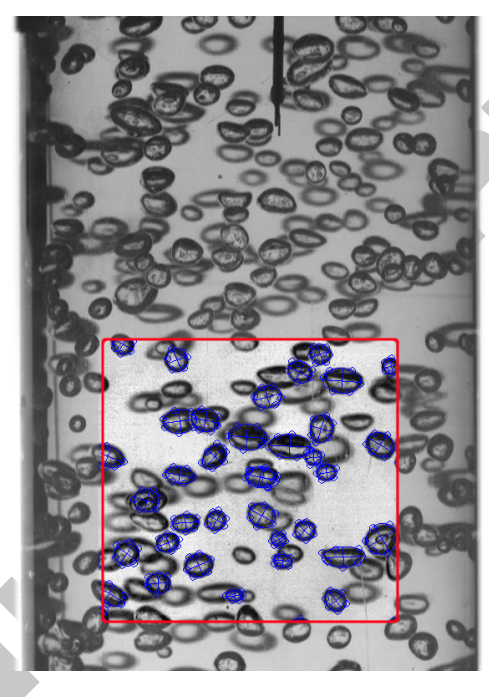

Figure 4: Example of some processed bubbles in an arbitrary region for an image frame in the lower measurement port.

Fig. 5 shows the BSD for the top measurement port. The BSD was fitted in the literature for bubbly flow to normal (Laakkonen et al., 2007), log-normal (Lage and Espósito, 1999; Parthasarathy and Ahmed, 1996; Ribeiro Jr. and Lage, 2004; Kazakis et al., 2008; Besagni et al., 2016), or gamma (Lim et al., 1990; Uga, 1972) distributions. For the experiments dealt in this paper, the bubble size data fitted well to a normal distribution. The Kolmogorov-Smirnov test at 5\% significance level was applied $(\mathrm{p}=0.79)$.

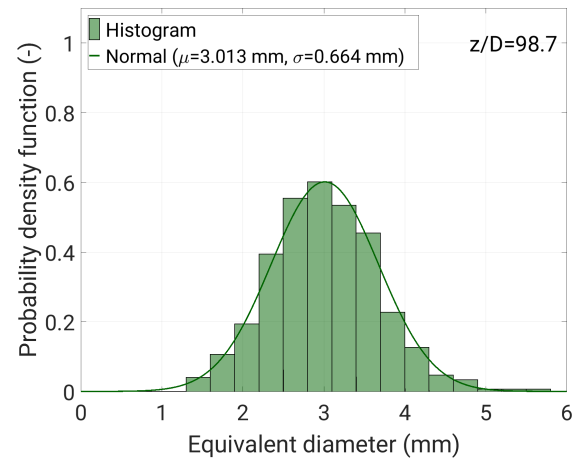

Figure 5: Bubble size distribution at the top measurement section (CFD outlet).

\subsection{Flow conditions and fluid properties}

This experimental facility was used to study in depth the condition shown in Table 1. Average data is obtained from measurement under this condition for different observations. The stable shows the superficial gas velocities, the statistical param35eters of the BSD at the inlet and the fluid temperature. 
Table 1: Flow conditions.

\begin{tabular}{lllll}
\hline $\begin{array}{l}j_{\mathrm{c}} \\
(\mathrm{m} / \mathrm{s})\end{array}$ & $\begin{array}{l}j_{\mathrm{d}} \\
(\mathrm{m} / \mathrm{s})\end{array}$ & $\begin{array}{l}\mu_{\text {inlet }} \\
(\mathrm{mm})\end{array}$ & $\begin{array}{l}\sigma_{\text {inlet }} \\
(\mathrm{mm})\end{array}$ & $\begin{array}{l}T \\
{[\mathrm{~K}]}\end{array}$ \\
\hline 0.5 & 0.03 & 2.76 & 0.64 & 293.15 \\
\hline
\end{tabular}

\section{Mathematical formulation and methods}

In this section we show the main formulation of the solver. It starts by introducing the DEM formulation for the disperse phase. Then, the Eulerian formulation for the continuous phase is summarized. Finally, the numerical methods employed and simulation setup are presented.

\subsection{DEM formulation}

The motion of the i-th bubble is computed by integrating the Newton's second law of motion:

$$
m_{i} \frac{\mathrm{d} \mathbf{u}_{\mathrm{b}_{i}}}{\mathrm{~d} t}=\mathbf{f}_{i}^{\mathrm{b}}+\mathbf{f}_{i}^{\mathrm{h}}+\sum_{j=1}^{\mathrm{IL}} \mathbf{f}_{i j}^{\mathrm{cf}} .
$$

In the left-hand side of this equation, $m$ stands for the bubble mass and $\mathbf{u}_{\mathrm{b}}$ for its instantaneous velocity. The first term on the right-hand side stands for the body force or buoyancy force due to the influence of the gravitational field on the bubbles:

$$
\mathbf{f}_{i}^{\mathrm{b}}=\mathrm{V}_{\mathrm{b}, \mathrm{i}} \mathbf{g}\left(\rho_{\mathrm{c}}-\rho_{\mathrm{b}}\right),
$$

being $V_{\mathrm{b}}$ the bubble volume, $\mathbf{g}$ the gravity vector, and $\rho_{\mathrm{c}}$ and $\rho_{\mathrm{b}}$ the carrier phase and bubble density. The bubble volume, is computed from the bubble diameter:

$$
V_{\mathrm{b}}=\frac{\pi d_{\mathrm{b}}^{3}}{6}
$$

The second term in Eq. 1 represents the hydrodynamic forces resulting from the liquid-bubble interaction as drag, lift, or virtual mass forces. The last term considers the collision forces between pairs of bubbles or between bubbles and walls. This is performed along the range of influence on each bubble with the data of bubbles or wall, stored in an interaction list (IL).

The hydrodynamic forces considered are the drag, lift, virtual mass and wall lubrication force:

$$
\begin{aligned}
& \mathbf{f}_{i}^{\mathrm{h}}=\frac{3}{4 d_{\mathrm{b}, \mathrm{i}}} \mathrm{V}_{\mathrm{b}, \mathrm{i}} \rho_{\mathrm{c}} \mathrm{C}_{\mathrm{d}, \mathrm{i}}\left(\mathbf{u}_{\mathrm{c}}-\mathbf{u}_{\mathrm{b}, \mathrm{i}}\right)\left|\mathbf{u}_{\mathrm{c}}-\mathbf{u}_{\mathrm{b}, \mathrm{i}}\right| \\
&+\mathrm{V}_{\mathrm{b}, \mathrm{i}} \rho_{\mathrm{c}} \mathrm{C}_{\mathrm{l}, \mathrm{i}}\left(\mathbf{u}_{\mathrm{c}}-\mathbf{u}_{\mathrm{b}, \mathrm{i}}\right) \times(\nabla \times\left.\mathbf{u}_{\mathrm{c}}\right)+\mathrm{V}_{\mathrm{b}, \mathrm{i}} \rho_{\mathrm{c}} \mathrm{C}_{\mathrm{v}, \mathrm{i}}\left(\frac{\mathrm{D} \mathbf{u}_{\mathrm{c}}}{\mathrm{D} t}-\frac{\mathrm{d} \mathbf{u}_{\mathrm{b}, \mathrm{i}}}{\mathrm{d} t}\right) \\
& \quad-\mathrm{V}_{\mathrm{b}, \mathrm{i}} \rho_{\mathrm{c}} \mathrm{C}_{\mathrm{w}, \mathrm{i}}\left|\mathbf{u}_{\mathrm{c}}-\mathbf{u}_{\mathrm{b}, \mathrm{i}}\right|^{2} \mathbf{n}_{\mathrm{w}} \cdot
\end{aligned}
$$

In this equation, $\mathrm{C}_{\mathrm{d}}, \mathrm{C}_{\mathrm{l}}, \mathrm{C}_{\mathrm{v}}$ and $\mathrm{C}_{\mathrm{w}}$ stand for the drag, lift, virtual mass and wall lubrication force coefficients. The instantaneous liquid velocity, $\mathbf{u}_{\mathrm{c}}$, used to compute the forces was calculated as the sum of the mean liquid velocity, $\mathbf{U}_{\mathrm{c}}$, and the ${ }_{431}$ fluctuating velocity component, $\mathbf{u}_{c}^{\prime}$. On the other hand $\mathbf{u}_{c}^{\prime}$ was ${ }_{432}$ computed with a Continuous Random Walk (CRW) stochastic ${ }_{433} 40$ cients are presented. model described later in Section 7 together with the turbulence modeling.

The contact forces are usually modeled with a soft-sphere model (Cundall and Strack, 1979) consisting of a spring, a dashpot and a slider. These need to define stiffness, as well as damping coefficient and friction coefficient. In this first approximation, viscous damping and tangential forces were not included in the analysis, so the force is restricted to normal collisions of a spring system. The force according to a linear contact-stiffness model gives:

$$
\mathbf{f}_{i j}^{\mathrm{cf}}=-k_{i j} \delta_{i j} \mathbf{n}_{\mathrm{w}}
$$

where $\delta_{i j}$ and $k_{i j}$ are the overlapping and stiffness respectively between two particles or between a particle and the wall.

Finally, after expanding and manipulating the Eq. 1 results:

$$
\begin{aligned}
& \left(m_{\mathrm{b}, \mathrm{i}}+\mathrm{V}_{\mathrm{b}, \mathrm{i}} \rho_{\mathrm{c}} \mathrm{C}_{\mathrm{v}, \mathrm{i}}\right) \frac{\mathrm{d} \mathbf{u}_{\mathrm{b}, \mathrm{i}}}{\mathrm{d} t}=\mathrm{V}_{\mathrm{b}, \mathrm{i}} \mathbf{g}\left(\rho_{\mathrm{b}}-\rho_{\mathrm{c}}\right) \\
+ & \frac{3}{4 d_{\mathrm{b}, \mathrm{i}}} \mathrm{V}_{\mathrm{b}, \mathrm{i}} \rho_{\mathrm{c}} \mathrm{C}_{\mathrm{d}, \mathrm{i}}\left(\mathbf{u}_{\mathrm{c}}-\mathbf{u}_{\mathrm{b}, \mathrm{i}}\right)\left|\mathbf{u}_{\mathrm{c}}-\mathbf{u}_{\mathrm{b}, \mathrm{i}}\right|+\mathrm{V}_{\mathrm{b}, \mathrm{i}} \rho_{\mathrm{c}} \mathrm{C}_{\mathrm{l}, \mathrm{i}}\left(\mathbf{u}_{\mathrm{c}}-\mathbf{u}_{\mathrm{b}, \mathrm{i}}\right) \times\left(\nabla \times \mathbf{u}_{\mathrm{c}}\right) \\
& +\mathrm{V}_{\mathrm{b}, \mathrm{i}} \rho_{\mathrm{c}} \mathrm{C}_{\mathrm{v}, \mathrm{i}} \frac{\mathrm{D} \mathbf{u}_{\mathrm{c}}}{\mathrm{D} t}-\mathrm{V}_{\mathrm{b}, \mathrm{i}} \rho_{\mathrm{c}} \mathrm{C}_{\mathrm{w}, \mathrm{i}}\left|\mathbf{u}_{\mathrm{c}}-\mathbf{u}_{\mathrm{b}, \mathrm{i}}\right|^{2} \mathbf{n}_{\mathrm{w}}-\sum_{j=1}^{\mathrm{IL}_{i}} k_{i j} \delta_{i j} \mathbf{n}_{\mathrm{w}} .
\end{aligned}
$$

\subsection{Eulerian formulation $(C F D)$}

The carrier phase motion is solved by the volume averaged Navier-Stokes equations for incompressible flow including the averaged properties of the disperse phase (disperse-continuous phase interaction and volume fraction). The local averaged continuity and momentum equations are (Kloss et al., 2012; Norouzi et al., 2016b):

$$
\frac{\partial}{\partial t} \alpha_{\mathrm{c}}+\nabla \cdot\left(\alpha_{\mathrm{c}} \mathbf{U}_{\mathrm{c}}\right)=0
$$

and

$$
\frac{\partial}{\partial t} \alpha_{\mathrm{c}} \mathbf{U}_{\mathrm{c}}+\nabla \cdot\left(\alpha_{\mathrm{c}} \mathbf{U}_{\mathrm{c}} \mathbf{U}_{\mathrm{c}}\right)=-\alpha_{\mathrm{c}} \nabla p+\nabla \cdot\left(\alpha_{\mathrm{c}} \mathbf{R}_{\mathrm{c}}\right)-\mathbf{M}^{\mathrm{h}},
$$

where $p$ is the density-normalized pressure, $\mathbf{M}^{\mathrm{h}}$ is the volumetric average of the interfacial forces and $\alpha_{\mathrm{c}}$ is the carrier phase volume fraction, generated by each bubble along its path on the cell. The method to calculate these coupling terms is described in Section 4. The Reynolds stress, $\mathbf{R}_{\mathrm{c}}$, is defined in Section 7.

\subsection{Particle force coefficients}

Eq. 6 shows the full force balance onto the i-th bubble. This equation depends on four coefficients that need to be specified to solve it. Next, the different correlations selected to model the interfacial coefficients and the definition of the stiffness coeffi- 


\subsubsection{Interfacial forces coefficients}

The drag coefficient of Tomiyama et al. (1998) for an airwater contaminated system reads:

$$
\mathrm{C}_{\mathrm{d}}, \infty=\max \left(\frac{24}{R e}\left(1+0.15 R e^{0.687}\right), \frac{8}{3} \frac{\mathrm{Eo}}{\mathrm{Eo}+4}\right) .
$$

This results in the drag coefficient curve of Fig. 6. The figure also shows the BSD at bottom and top ports. In the simulation, the drag coefficient will influence the individual velocity of each bubble. According to the nonlinearity of the drag force, individual velocity of each bubble will be calculated. Note that changes in size from the inlet to the top of the pipe can influence variables related with the bubble velocity as the volume fraction.

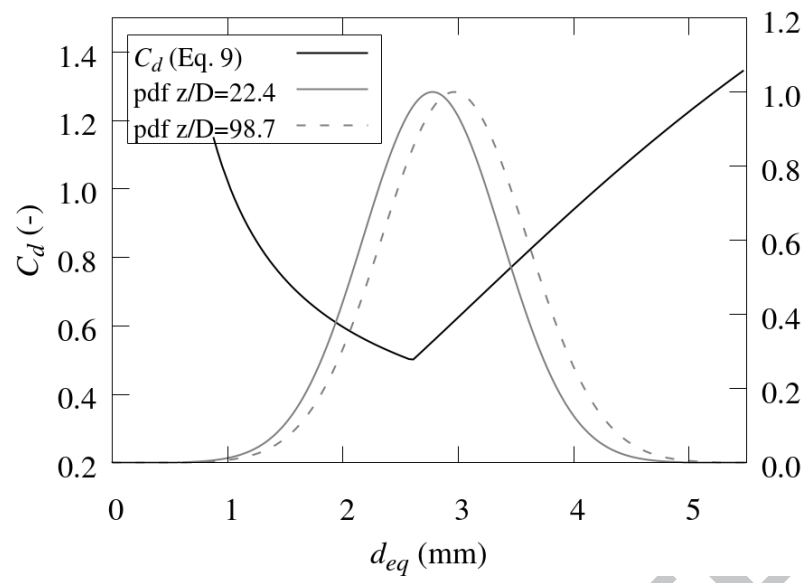

Figure 6: Drag coefficient and bubble size distribution at bottom and top ports.

The previous drag coefficient was obtained for experiments performed in a stagnant liquid. In the literature an increase on the drag coefficient as a function of the strain rate, Sr, was reported (Legendre and Magnaudet, 1998; Magnaudet and Eames, 2000; Sugioka and Komori, 2009). The relation found by Legendre and Magnaudet (1998) is used to describe the drag force:

$$
C_{\mathrm{d}}=C_{\mathrm{d}, \infty}\left(1+0.55 \mathrm{Sr}^{2}\right), \quad \mathrm{Sr}=\frac{d_{\mathrm{b}} G}{\left|\mathbf{u}_{\mathrm{b}}-\mathbf{u}_{\mathrm{c}}\right|},
$$

where $G$ is the magnitude of the carrier phase velocity gradient.

The lift coefficient of Tomiyama et al. (2002) was used to ${ }_{481}$ consider the influence of the shear rate for the different bubble sizes present in the simulations:

$$
C_{1}= \begin{cases}\min (0.288 \tanh (0.121 R e), f) & \mathrm{Eo}_{d}<4 \\ f_{l} & 4 \leq \mathrm{Eo}_{d} \leq 10 \\ -0.29 & \mathrm{Eo}_{d}>10\end{cases}
$$

and

$$
f_{l}=0.00105 \mathrm{Eo}_{d}^{3}-0.0159 \mathrm{Eo}_{d}^{2}-0.0204 \mathrm{Eo}_{d}+0.474
$$

where $\mathrm{Eo}_{d}$ is a modified Eötvös number with characteristic length the maximum horizontal dimension of the bubble. It is estimated in Tomiyama et al. (2002) using the empirical correlation for the aspect ratio of Wellek et al. (1966). In the literature, recent studies (Cai et al., 2010; Legendre et al., 2012) and different correlations (Yamoah et al., 2015; Besagni and Inzoli, 2016) related with the aspect ratio of bubbles can be found. The latter two correlations and the Wellek's one give positive values for the lift coefficients for the BSD of this work. In this study the Wellek's correlation is used, the lift coefficient related with the BSD is shown in Fig 7.

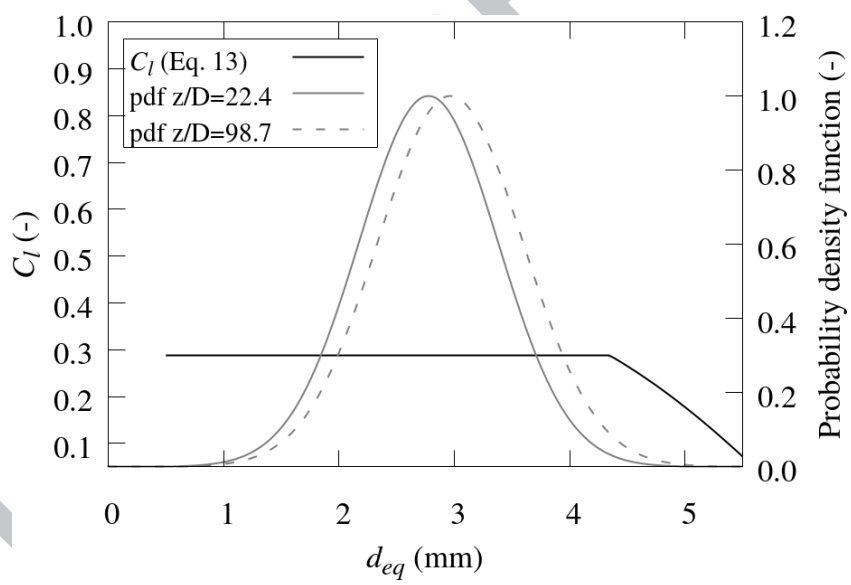

Figure 7: Lift coefficient and bubble size distribution at bottom and top ports.

The wall lubrication force (Antal et al., 1991) is a hydrodynamic force usually taken into account to simulate two-phase flow in pipes. This force reflects the drainage of the fluid around the bubble and represents the force that the liquid drainage around a bubble moving near a wall has on the bubble. A twodimensional solution was derived for flow between a cylinder and a wall by Antal et al. (1991). The constants obtained in this work were evaluated by a 3D DNS of viscous flow past a single bubble with uniform velocity using PHOENICS code. The simulations were done for two relative velocities $(0.1$ and $0.2 \mathrm{~m} \mathrm{~s}^{-1}$ ) finding the following equation:

$$
\mathrm{C}_{\mathrm{w}}=\max \left[0, \mathrm{C}_{\mathrm{w} 1}+\mathrm{C}_{\mathrm{w} 2} \frac{R_{b}}{y}\right],
$$

where $y$ is the distance of the bubble center to the wall. The fitting coefficients can be expressed as:

$$
\mathrm{C}_{\mathrm{w} 1}=-0.06\left|\mathbf{u}_{\mathrm{c}}-\mathbf{u}_{\mathrm{b}, \mathrm{i}}\right|-0.014,
$$

and

$$
\mathrm{C}_{\mathrm{w} 2}=0.147 \text {. }
$$

In Fig. 8 we show this coefficient for three different bubble diameters. In the figure, the dots show where the distance to the wall is equivalent to the bubble radius. This point represents the limit where the bubble is in contact with the wall. Dashed lines are plotted for distances to the wall to the left of this point. As 
we use a discrete element method, the bubble center is determined mainly by the bubble-wall contact fore, then the coefficient would fall around the range represented by the solid lines. Note, that if the same model is applied for TFM this may result in an overestimated force.

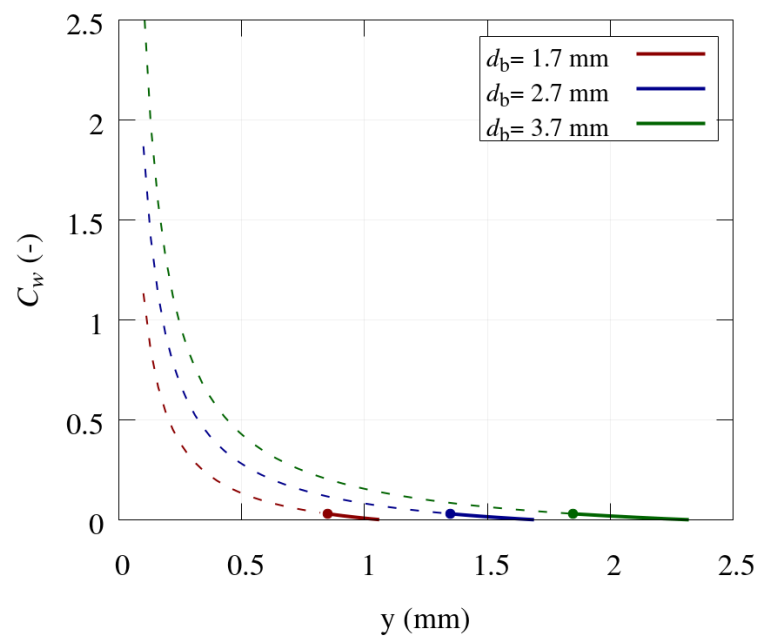

Figure 8: Wall lubrication force coefficient for three different bubble diameters assuming a relative velocity of $0.2 \mathrm{~m} \mathrm{~s}^{-1}$.

Finally, applying potential flow theory to flow around a

We summarize the different interfacial forces models used in this work in Table 2.

Table 2: Coefficients for interfacial force closures.

\begin{tabular}{ll}
\hline Drag force & Tomiyama et al. (1998) \\
Lift force & Tomiyama et al. (2002) \\
Virtual mass force & Drew and Lahey (1987) \\
Wall lubrication force & Antal et al. (1991) \\
\hline
\end{tabular}

\subsubsection{Stiffness coefficient}

The stiffness of the bubbles were determined as a function of the surface area increase due to the bubble deformation. Assuming that in the deformation the bubble conserves the volume, the surface energy changes because of surface deformation, $\Delta A$, for a given surface tension, $\gamma$. To calculate the stiffness it is assumed that the bubbles have a spherical shape unless they are colliding. Once they collide, their shape deforms to an oblate spheroid with its minor-axis along the collision direction (see Fig. 9).

We assume that $a$ and $b$ are the semi-minor axis and semimajor axis of the $\mathrm{i}$-th and j-th bubbles. The bubble deformation is accounted for by the normal overlapping defined as:

$$
\delta_{i j}=0.5\left(d_{b, i}+d_{b, j}\right)-l_{i j},
$$

where $l_{i j}$ is the distance between the bubble centers. The semi-axis $a_{i}$ is defined as $a_{i}=r_{b, i}-0.5 \delta_{i j}$. The semi-axis b results549

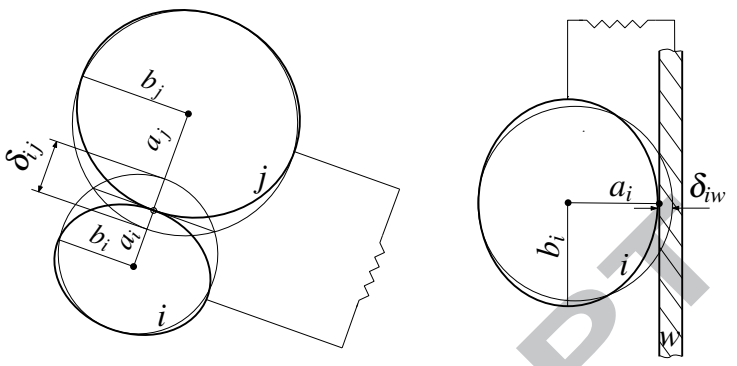

Figure 9: Mass-spring system for the soft-sphere model.

in $b_{i}=\sqrt{r_{b, i}^{3} / a_{i}}$.

Combining the works done by the bubble deformation and the spring system (Eq. 5) it results:

$$
\int_{0}^{\delta_{i j}} k_{i j} \delta_{i j} d \delta_{i j}=\gamma\left(\Delta A_{i}+\Delta A_{j}\right)
$$

Thus, the stiffness between the two bubbles can be calculated as:

$$
k_{i j}=\frac{2 \gamma\left(\Delta A_{i}+\Delta A_{j}\right)}{\delta_{i j}^{2}}
$$

The value of $k_{i j}$ is calculated dynamically for each bubble movement if the bubble is interacting with other bubbles or walls. The wall is considered as a rigid body, $j$ index is replaced by $w$ in the above equations for solving the overlapping of the bubbles with the wall. 497

\subsection{Numerical methods and simulation set up}

A CFD-DEM solver to simulate bubbly flow was developed using the OpenFOAM ${ }^{\circledR}$ tools and methods. As a starting point the Lagrangian libraries of OpenFOAM ${ }^{\circledR}$ (mainly the basic and intermediate) are considered to create a new bubblyFlow library and a new bubbleCFDEM solver. It started from the version 2.3.x and incorporates the improvements from subsequent versions. The OpenFOAM's modular structure was followed to allow future contributions of submodels and methods.

499 The solution procedure consists on solving the CFD and ${ }^{50}$ DEM parts explicitly. Thus, the pressure-velocity calculation ${ }^{50}$ procedure for the Navier-Stokes equations is solved first. Then ${ }^{50}$ the bubbles are tracked during a given number of subcycles un${ }^{50}$ til the Eulerian time step, $\Delta t$, is reached. During the tracking, in ${ }^{504}$ each substep, $\mathbf{u}_{\mathrm{c}}^{\prime}$ is computed from the CRW stochastic model. ${ }^{50} \mathbf{U}_{\mathrm{c}}$ is updated according to the interpolated values at the current ${ }^{506}$ position of the bubble to obtain $\mathbf{u}_{\mathrm{c}}=\mathbf{U}_{\mathrm{c}}+\mathbf{u}_{\mathrm{c}}^{\prime}$, needed to calcu${ }^{50}$ late the hydrodynamic forces and the new bubble velocity. The ${ }^{508}$ coupling terms are calculated averaged on time during the path ${ }^{50}$ to provide $\mathbf{M}^{\mathrm{h}}$ and $\alpha_{\mathrm{c}}$ to solve pressure and velocity in the next ${ }^{510}$ steps. In each substep the bubble radius $R_{b}$ is updated to con${ }^{511}$ sider the bubble size changes due to the pressure according to Eq. 27 a simplified model.

Eq. 8 and Eq. 7 were solved with the Pressure-Implicit with ${ }_{51}$ Splitting of Operators (PISO) algorithm (Issa, 1986) includ51ing the volume fraction and momentum exchange. Eq. 28 and 
Eq. 30 defining the turbulence model are incorporated in the algorithm and will be discussed later.

A modified version of the tracking algorithm of Macpherson et al. (2009) was developed and implemented in the solver to incorporate the modifications described in this work. A secondorder leapfrog integrator was used to numerically solve Eq. 6, alternating a linear move and collision in time. This integrator is symplectic and the energy error remains bounded for sufficiently small time steps. The collisions are performed using the Arbitrary Interaction Cells Algorithm (AICA) already implemented in OpenFOAM ${ }^{\circledR}$ (Macpherson and Reese, 2008). With this algorithm the particle-particle and particle-wall interactions are evaluated for the bubbles in the given referred cells. The referred cells are defined at the beginning of the simulation according to a specified $r_{c u t}$ value, larger than the maximum bubble radius expected. The soft-sphere model is then applied for the bubbles that are overlapping.

The pipe length between bottom and top measurement ports is considered for the model. The mesh was created by the blockMesh tool of OpenFOAM ${ }^{\circledR}$, resulting in an unstructured hexahedral mesh with an O-grid structure. The mesh is defined by an axial mesh size of 1.5 times the maximum bubble diameter considered. The number of elements of the mesh results in 209280. The mesh is shown in Fig. 10. Appendix A shows a mesh sensitivity study in which the mesh density is examined with three configurations.

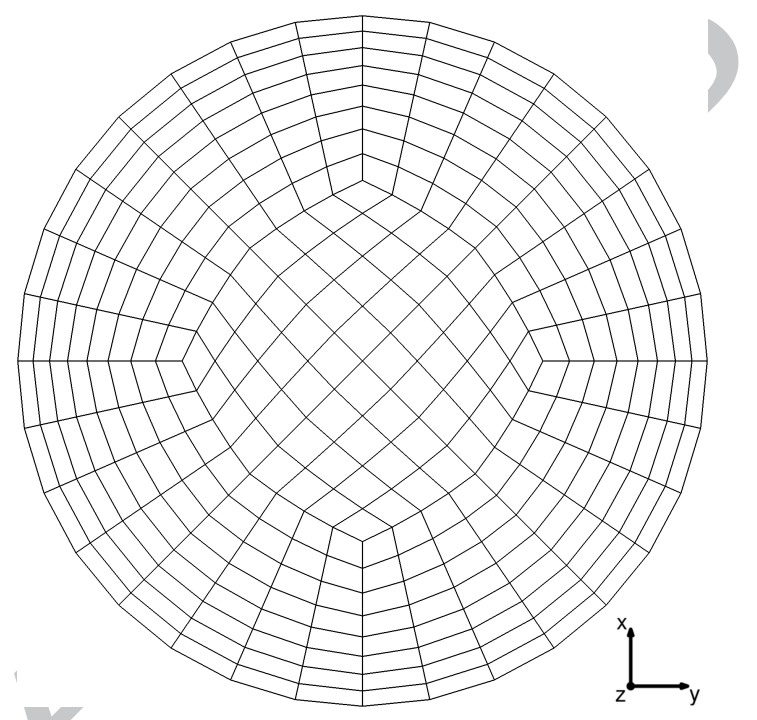

Figure 10: Cross-section mesh view.

The time step for this simulation is defined according to the restrictions of both phases. For the continuous phase, a low Courant number must be fixed to guarantee stability, especially for a transient simulation and although an implicit solver is used (Jasak, 1996; Norouzi et al., 2016d). The value of $\Delta t$ selected for the simulation is fixed to a value of $7.5 \times 10^{-4} \mathrm{~s}$ giving a maximum Courant number of around 0.2.

The time step for the DEM part is given by the specified number of subcycles. It has to be smaller than the particle response time to be able to capture the changes in the local flow velocity tion, the time step should be sufficiently small to capture the bubble collision interaction to avoid inaccurate or unstable simulations. This is usually related to the oscillation period of the spring-mass system (Tsuji et al., 1993):

$$
\lambda=2 \pi \sqrt{m / k}
$$

At least one-tenth of this value is required for the time step for integrating the equations of motion (Tsuji et al., 1993; Norouzi et al., 2016c). As the stiffness is computed dynamically for the bubbles we need to define a sufficiently small timestep. Accordingly, a value of 10 subcycles results in a time step of $7.5 \times 10^{-5}$ s satisfying this criteria.

The simulation was run until a total time of $35 \mathrm{~s}$ was reached. The averaging of the results starts after the first $5 \mathrm{~s}$ to obtain the averaging over $30 \mathrm{~s}$ as in the real experiments. The simulation was run with an Intel(R) Xeon(R) CPU E5-2450 @2.10GHz using a single processor, requiring a wall-clock time of around 15 days.

The boundary conditions used in the simulations are listed in Table 3. The inlet boundary condition established as a fixed value has the experimental profile values obtained at the measurement port $\mathrm{z} / \mathrm{D}=22.4$.

Table 3: Boundary conditions for inlet, outlet and wall patches.

\begin{tabular}{clll}
\hline Variable & Inlet & Outlet & Wall \\
\hline $\mathbf{U}_{\mathrm{c}}$ & Dirichlet & Neumann & No-slip \\
$\mathrm{p}$ & Neumann & Dirichlet & Neumann \\
$\kappa_{\mathrm{c}}$ & Dirichlet & Neumann & wall function \\
$\varepsilon_{\mathrm{c}}$ & Dirichlet & Neumann & wall function \\
$v_{t, \mathrm{c}}$ & - & - & wall function \\
\hline${ }^{*}$ zero gradient & & \\
$\dagger$ Troshko and Hassan $(2001 \mathrm{a}, \mathrm{b})$ &
\end{tabular}

\section{Void fraction and momentum exchange assignment}

The bubble volume and momentum exchange assignment is important for the coupling between the phases as determines the velocity-pressure results and the solver stability. Furthermore, it determines the local values of the bubble forces. Therefore, an efficient consideration of the local void fraction and momentum exchange becomes mandatory.

From the pioneering work of Crowe et al. (1977) who accounted for the mass, momentum, and energy coupling between phases through the Particle-Source-In Cell (PSI) model, several methods have been proposed. According to Norouzi et al. (2016d) the void fraction, and therefore the momentum term, in a cell can be calculated by exact analytical (Wu et al., 2009b; Peng et al., 2014; Wu et al., 2009a) or approximate nonanalytical methods. Among the second type, they can be classified as Particle Center Method (PCM) (Xu and Yu, 1997), ${ }_{58}$ porous cubes (Deen et al., 2004; Link et al., 2005), statistical 58approaches (Xiao and Sun, 2011), subelement (Gui et al., 2008; ${ }_{58}$ Hilton et al., 2010; Norouzi et al., 2016a) and spherical control 580volume (Kuang et al., 2008). The first type of methods has the 
advantage of being accurate but hardly suitable for non-regular meshes. Therefore, the nonanalytical approach would be most effective for this case. These techniques usually gives satisfactory results at the expense of computational time.

The algorithm shown in Vaidya et al. (2006); Macpherson et al. (2009) is implemented in OpenFOAM ${ }^{\circledR}$ to track particles along the cells. The motion was done for unstructured, arbitrary polyhedral meshes leading with 3D meshes of complex geometries. The assignment of void fraction and momentum exchange during the tracking is based on the PCM. This gives satisfactory results for scenarios where the cell volumes are regular and much larger than the volume occupied by the particles. In the PCM method all the bubble volume is assigned to the cell where the particle center is located. As reported by Peng et al. (2014) it may lead to an error up to $50 \%$ when the particle center is near the cell boundaries or to numerical instabilities due to dramatic changes in void fraction. The subelement method is used to improve precision and accuracy. Dividing the particle in a given number of elements each subelement contributes its volume to the cell where the subelement center is located during its path.

\subsection{Description of the new equivolumetric subelement method}

Similarly to the subelement method, we have developed an equivolumetric subelement method to track a fixed number of representative volumes or subelements of each bubble. To produce an optimal distribution we divide the sphere with the equivolumetric partitioning algorithm defined in Yang and Chen (2006). The method is performed in three main steps. First, an initial division of the sphere into two solid hemispheres is performed. Later each semi-sphere is divided into $\mathrm{n}$ hemispherical shells of equal thickness $\delta r=r_{b} / n$ (note the first shell results in a small hemisphere). Finally, in the last step, the basic elements are generated from the hemispherical shells. An example of the $\mathrm{i}$-th shell partitioning is shown for a radius $\pi$ in Fig. 11.

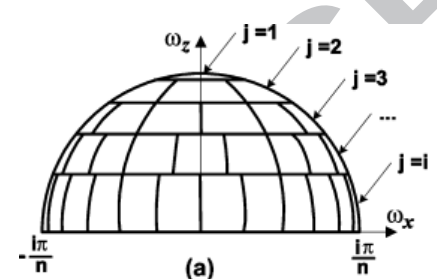

(a)

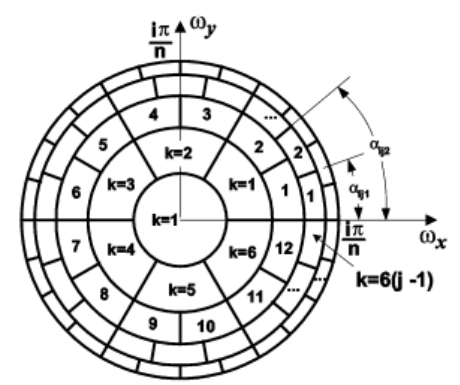

(c)
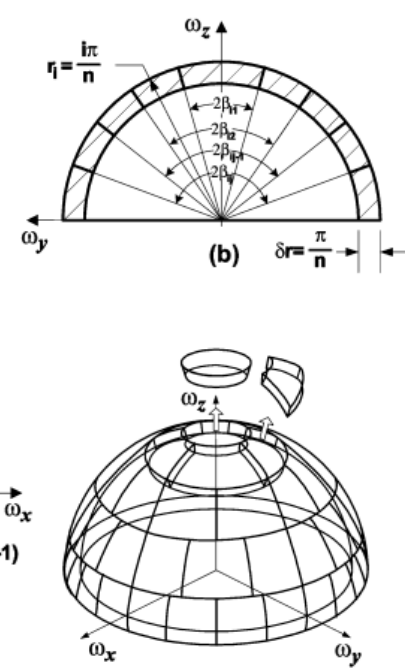

(d)

Figure 11: Equivolumetric and uniform convergent partition of hemispherical 683 shell Yang and Chen (2006).

The i-th shell is sliced into i polar divisions, and labeled by index $j=1,2, \ldots, i$. Then every polar slice is cut into pieces given by $\mathrm{k}=1,2, \ldots, 6(\mathrm{j}-1)$. According to this method, a sphere is partitioned exactly into a $2 n^{3}$ cuts of equivalent volume depending on the number of $\mathrm{n}$ shells specified for each sphere.

The angles $\beta_{i j}$ and $\alpha_{i j k}$ described in Fig. 11 determine the polar and azimuthal coordinates of the resulting cuts:

$$
\begin{gathered}
\alpha_{i j k}=\frac{2 k \pi}{6(j-1)} \\
\beta_{i j}=\cos ^{-1}\left(\frac{3(i-j)(i+j-1)}{1+3 i(i-1)}\right)
\end{gathered}
$$

To apply this equivolumetric partition of the bubble to the simulation, the bubbles are divided dynamically when they are seeded. A common $\delta r$ is fixed for all the bubbles and the bubbles are partitioned according to the number of shells obtained ( $\left.n=R_{b} / \delta r\right)$ and applying a ceiling function (next largest integer). Figure 12 shows an example for a bubble of $2.5 \mathrm{~mm}$ radius divided by 3 shells. The residence time of each element in the cells is computed to determine the contribution of momentum and volume fraction into the grid.

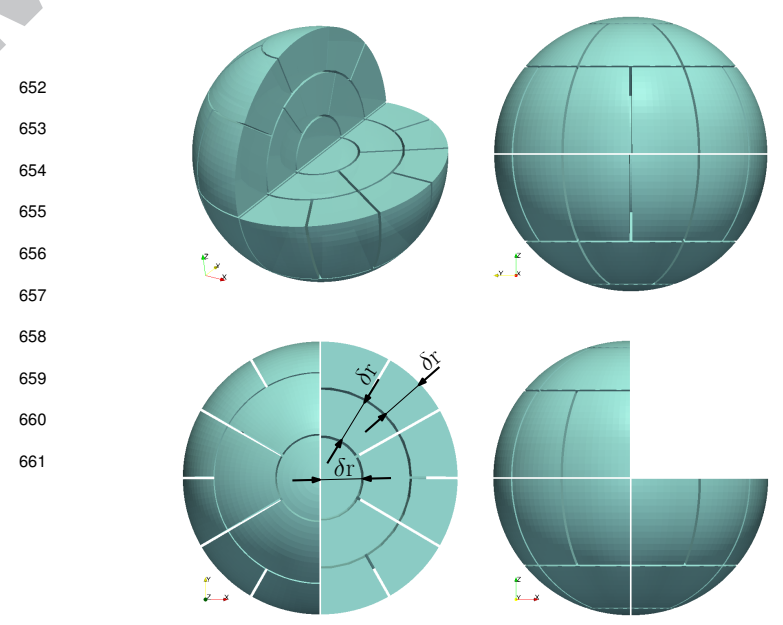

Figure 12: Views of the equivolumetric partition for a bubble of diameter 2.5 $\mathrm{mm}$.

Considering that many bubbles may be present in a cell, the exchange momentum in a cell for a given subcycle can be calculated as:

$$
\mathbf{M}^{\mathrm{h}}=\frac{1}{\rho_{\mathrm{c}} V_{\text {cell }}} \sum_{i=1}^{N_{b}} \sum_{j=1}^{N_{c s, i}} \frac{\mathbf{f}_{i}^{\mathrm{h}}}{N_{s, i}}
$$

where $V_{\text {cell }}$ is the cell volume, $N_{b}$ the number of bubbles entirely or partialy in the cell, $N_{c s, i}$ the number of subelements belonging to the i-th bubble inside the cell, and $N_{s, i}$ the number of subelements that compose the i-th bubble. 
The carrier phase volume fraction is calculated in the same way as:

$$
\alpha_{\mathrm{c}}=1-\frac{1}{V_{\text {cell }}} \sum_{i=1}^{N_{b}} \sum_{j=1}^{N_{c s i}} \frac{\mathrm{V}_{\mathrm{b}, \mathrm{i}}}{N_{c s, i}}
$$

\subsection{Validation and convergence study}

To be valid, in the unresolved CFD-DEM method the volume cell must be larger than that of the particles. The cases under investigation in the present work are highly restrictive according to the diameter pipe and bubble size. This means that the mesh size should be sufficiently small to capture the hydrodynamics on the pipe and sufficiently large to satisfy the requirements of the method. Employing the $\delta r$ parameter defined before, we studied the maximum ratio of total volume occupied by the bubbles and volume cell (or void fraction) obtained at each time step. This suggest the worst-case scenario where, for instance, the smallest cell volume concurs with the biggest bubble. This has been checked for the three conditions shown in this paper. Figure 13 shows the probability density function of the maximum local void fraction for different values of $\delta r$.

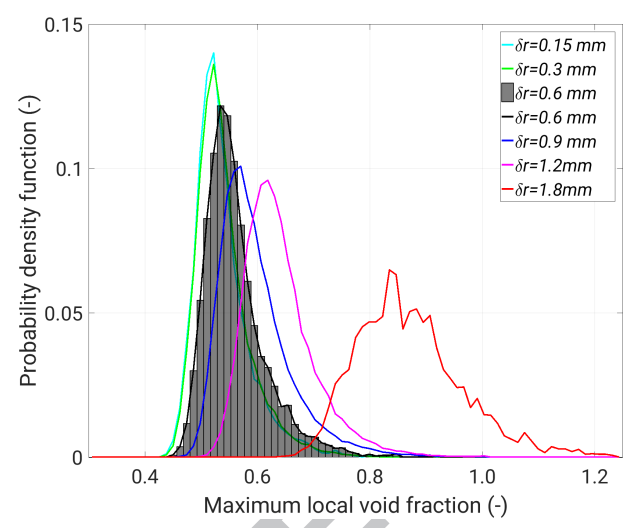

Figure 13: Maximum local void fraction among the time steps for different $\delta r$ values.

The results suggest that the maximum local void fraction decrease as the value of $\delta r$ is decreased, until reaching minimum values. For $\delta r=1.8 \mathrm{~mm}$, maximum void fraction values above 1 were obtained in the domain. In fact, this caused convergence problems stopping the simulation. This was observed for other tests of $\delta r$ from this value. We also analyzed the special situation where $\delta r$ is sufficiently large to result in only a subelement per bubble, which would be equivalent to a PCM method. These simulations stopped earlier and stability problems related to the unrealistic changes in time of local void fractions were detected. In contrast, small values of $\delta r$ results in greater number of subelements. This allows a more accurate assignment of bubble volume into the cells, improving the convergence and the accuracy of the pressure and velocity fields calculation. The wall-clock time for $\delta r=0.3 \mathrm{~mm}$ was around four times higher than for $\delta r=0.6 \mathrm{~mm}$. Note that the number of elements increase cubically with $\delta r$. Then, a value of $\delta r=0.6 \mathrm{~mm}$ was used for convenience for this simulation, as it gives a good balance between computational time and performance.

To verify the void fraction assignment method, we compare the time-averaged values of the cell interpolated values with the void fraction at probe locations as the latter are meshindependent (Fig. 14). The figure shows a good agreement between the local definition of void fraction with the values obtained from the contribution of the bubbles into the grid.

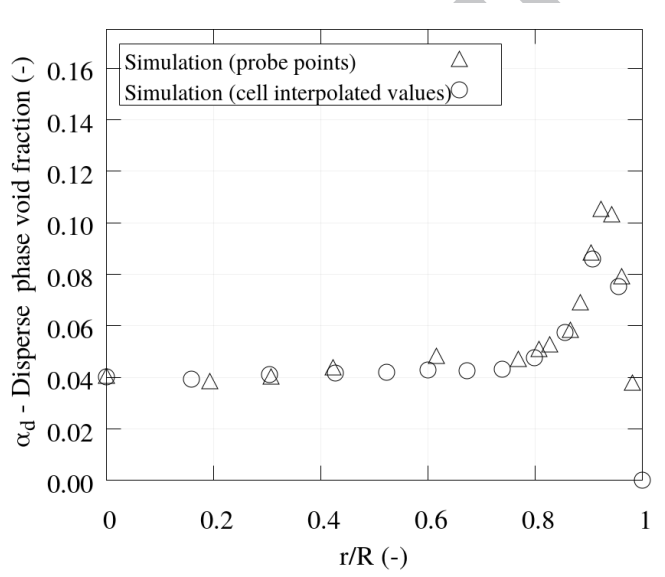

Figure 14: Cell interpolated values of void fraction for $\delta r=0.6 \mathrm{~mm}$ compared with void fraction at probe points.

\section{Seeding model and fluid flow influence}

The position in which the bubbles are injected in the domain determines the disperse phase evolution because of the local momentum exchange from the bubbles to the fluid. In fact, the void fraction and bubble density radial profiles should be in accordance to the experiments at the inlet. In the present simulations the bubbles seeding over time must be done under these time-averaged values constraints. In this section we propose an algorithm for the random-polydisperse seeding of bubbles in circular pipes. This accomplishes with the statistical properties of given BSD, void fraction and bubble frequency profiles. This algorithm can be easily extended to include more constraints if ${ }_{703}^{702}$ needed.

${ }_{704}^{703}$ To show the strength of the proposed algorithm, we make use ${ }_{705}^{704}$ of it to study the effects of bubble seeding distribution on flow ${ }^{705}$ evolution. Thus, the flow characteristics and evolution have 706
707 ${ }_{707}^{70}$ addition to the pattern with a profile from the bubble frequency ${ }_{709}^{708}$ of the experiments, a uniform distribution, and random radius ${ }_{710}^{709}$ and polar angle will be studied (see Fig. 15).

711

\section{${ }_{712}^{711}$ 5.1. Seeding algorithm to seed the bubbles}

713 A circular section of radius $R_{p}$ in polar coordinates with $r$ ${ }_{71}$ (radial coordinate) and $\theta$ (angular coordinate) is considered. A 7 aniform distribution of seeding points over $\left[0, R_{p}\right]$ and $[0,2 \pi]$ 71tespectively, would give an accumulation of bubbles near the 71center as the area is proportional to the squared distance of $r$ 7to the center. To get an area-uniform random distribution, the 


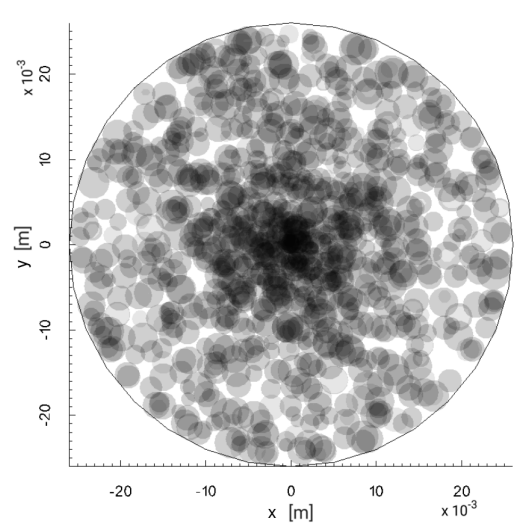

(a) Random $r$ and $\theta$

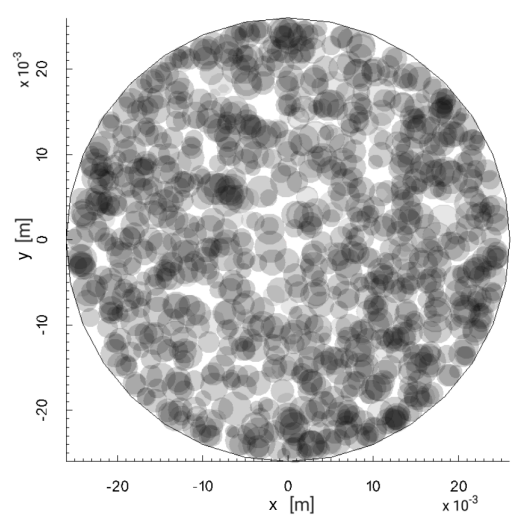

(b) Unfiformly distributed

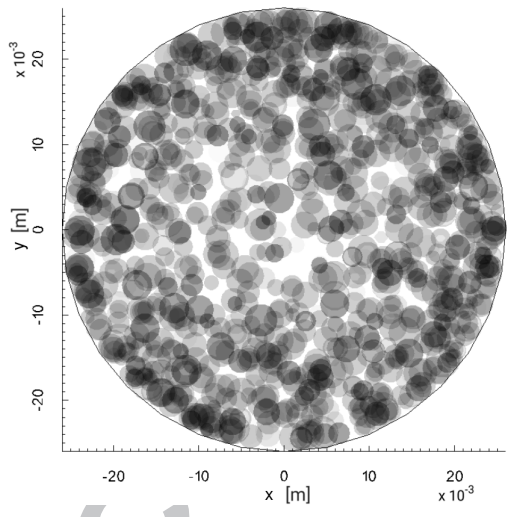

(c) Profile from experiments

Figure 15: Injection patterns considered. Bubbles at injection during $1 \mathrm{~s}$ with a sampling period of $0.075 \mathrm{~s}$.

probability density function of the random variable should be $f(r)=2 r / R_{p}^{2}$. Instead of a uniform distribution, it is more convenient to seed the bubbles according to a given radial condition. For a given experimental sample of bubbles, if we know the radial distribution of bubbles detected per unit time the probability density function can be expressed as:

$$
f(r)=n_{f}(r) \frac{2 r}{R_{p}^{2}}
$$

Based on $f(r)$ we developed the algorithm shown in Fig. 16 to seed the bubbles in the system following a BSD in a corresponding polar position vector $\mathbf{r}$ with random radial, $\xi_{r}$, and angular, $\xi_{\theta}$, positions.

This algorithm provides the seeding of bubbles during a given seeding time $t_{i}$. An average time step volume, $\mathrm{V}_{i}$, is defined at the beginning of the simulation from a given air volumetric flow rate, $Q_{\mathrm{g}}$, and $\Delta t$. A volume $\mathrm{V}_{t}$ is calculated at each Eulerian time step. Bubbles with a size from a given BSD are consecutively generated until the total volume of these bubbles reach $\mathrm{V}_{t}$. A deviation between $\mathrm{V}_{i}$ and $\mathrm{V}_{t}$ is expected as each bubble size is obtained randomly from the BSD. Then, the volume $\mathrm{V}_{t}$ doesn't match $\mathrm{V}_{i}$ accurately, especially for low time steps. A correction variable, $\mathrm{dV}$, is introduced in the algorithm to store the exceeding or missing volume for a given time step, to correct $\mathrm{V}_{t}$ in the next time step.

The radial coordinate is randomly obtained from the defined $f(r)$, while the angular one is randomly uniformly distributed. The geometrical constraints of the bubble sizes and the pipe wall are considered in order to avoid unrealistic overlapping between two bubbles or with the wall. Each time-step a bubble can be discarded if overlaps another bubble previously seeded close to it. In order to speed up the algorithm, an iterative process to try the seeding at different random azimuthal positions for a given radial position is performed until a maximum number of tries (iMax), predefined by the user.

The discarded bubbles are stored in a list to try its seeding in the next time step. This step is necessary to keep the statistic consistent over time. Position and diameters are stored in a790 data list where the First-In, First-Out (FIFO) rule is applied to791

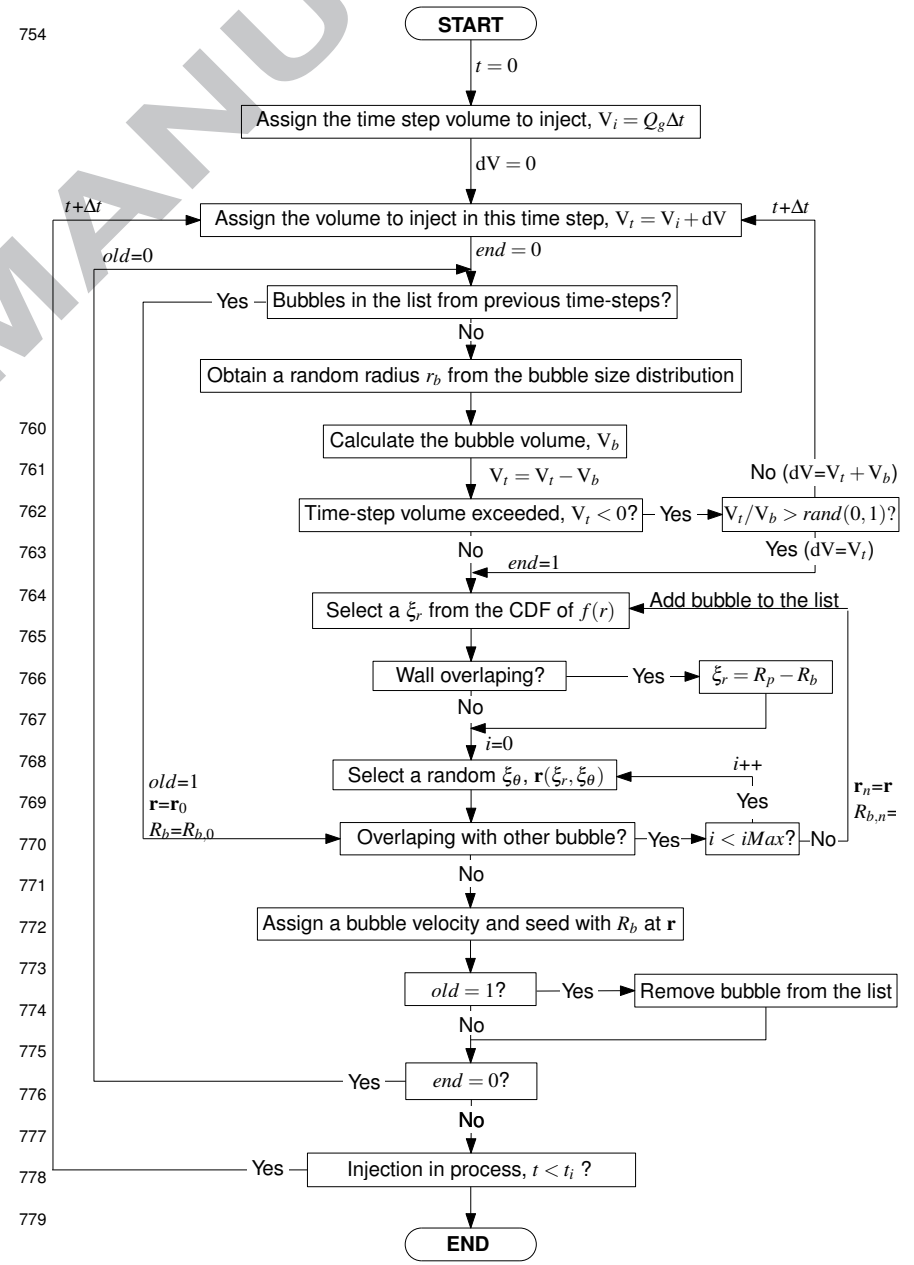

Figure 16: Seeding algorithm diagram.

try first the seed of the oldest bubbles in the list. In Fig. 16, the index $n$ refers to the tail of the list and the index 0 to the head. 


\subsection{Boundary conditions and flow influence of the seeding pat-} terns

In this subsection we analyze the use of the seeding algorithm. The bubbles are introduced in the system according to the experimental data specified by the air flow rate. To check the injected volume, we show in Fig. 17 the superficial gas velocity over time.

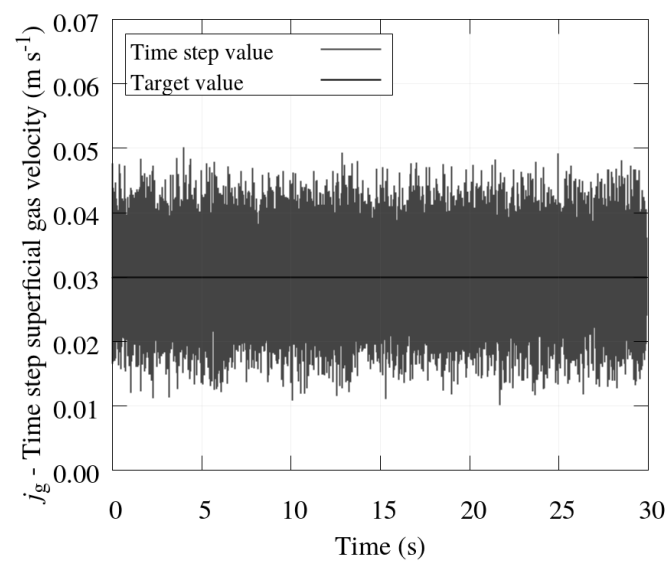

Figure 17: Superficial gas velocity at inlet in the simulation compared with the target value $j_{\mathrm{g}}=0.03 \mathrm{~m} \mathrm{~s}^{-1}$.

The inlet superficial gas velocity in the simulation was calculated from the volume injected at each Eulerian time step. This is compared with the target value specified in the experiments from the air flowmeter. The average of the time step values verifies that the seeding process in the simulation was performed properly.

Furthermore, a deviation is expected in the experiments be-

tween the air flow rate given by the flowmeter and the measured 831806 The three seeding patterns shown in Fig. 15 were used to by the probes. This should be considered when analyzing the 322 8о analyze the axial evolution of the carrier phase velocity and computational results. The air flow rate measured at the probes,, 833 $808 \mathrm{~g}$ (oid fraction at the center of the pipe $(\mathrm{r} / \mathrm{R}=0)$ and near the wall $Q_{N P S}$, is compared with air flow rate given by the flowmeter,834 $\left.80 \mathrm{r} / \mathrm{R}=0.94\right)$. In Fig. 19 we note how the velocity is affected by $Q_{F M}$. Note that the former is obtained from the cross-sectional835 810 the bubbles as expected, especially for the random $\mathrm{r}$ and $\theta$ posiaveraged $\alpha_{\mathrm{d}}$ and $V_{z}$. The resulting value of $Q_{N P S}$ is around 7\%836 ${ }_{81}$ tion pattern.

lower than $Q_{F M}$. This value is in accordance to the findings of 837812 The simulations show that depending on the seeding pattern, Barrau et al. (1999) who noticed errors around -0.8 and $-16 \%$ 838 st the evolution of the flow is different. The influence of the seedcompared with the flowmeter reference measurements. This er-8з9 siang is smoothed as the flow advances and eventually lost after rors were attributed to the bubble-probe interaction. The choice 840 819 certain evolution distance for this case. For this particular of $Q_{F M}$ as the boundary condition for the simulation provides a 841 s1experimental facility, the mid-port location is set at $\mathrm{z} / \mathrm{D}=61.0$, more accurate gas flow rate in the system. It is needed to prop-842 ${ }_{1180}$ so for the validation at this height the results of the simulaerly account for the coupling effects affecting to the velocity 843 81tion would not depend on the seeding. There may be situations and turbulence liquid phase and indirectly to the disperse phase. 844 $81 \mathrm{wh}$ here the seeding has a strong impact when performing an exHowever, the comparison of the resulting void fraction profiles 845 82perimental validation of the solver. This will depend on the will show discrepancies that are given for the uncertainties of ${ }_{846}{ }_{82} \mathrm{gas}$ and liquid velocities and the height where the validation is the measurements.

84782 performed. If the comparison with experiments is done at a dis-

The data measured at $\mathrm{z} / \mathrm{D}=22.4$ is considered for the inlet 848 82 2 ance close to the inlet, important discrepancies can be found. of our simulation, so the experimental measures of $n_{f}$ are used ${ }_{849}{ }_{82}$ However, it may not be known in advance the flow evolution for to estimate $f(r)$. In Fig. 18 we compare the experimental datasso 82 any flow condition or configuration. Then, the validation could and the results of the simulation immediately after the seeding.851 $82 \mathrm{be}$ affected by the inlet. Also, note that for simulations with The comparison for $n_{f}$ and $\alpha_{\mathrm{d}}$ profiles shows that the seeding ${ }_{852}$ 82 $^{2}$ oalescence or breakup it could lead to different axial evolution was performed representing properly the experimental data pro-853 vided. Higher void fraction profiles are appreciated because of 854 the differences between $Q_{N P S}$ and $Q_{F M}$. as an inaccurate seeding algorithm would lead to unrealistic coalescence and breakup rates. The proposed algorithm provides 855 a generic injection for a nonuniform size inlet to represent the 

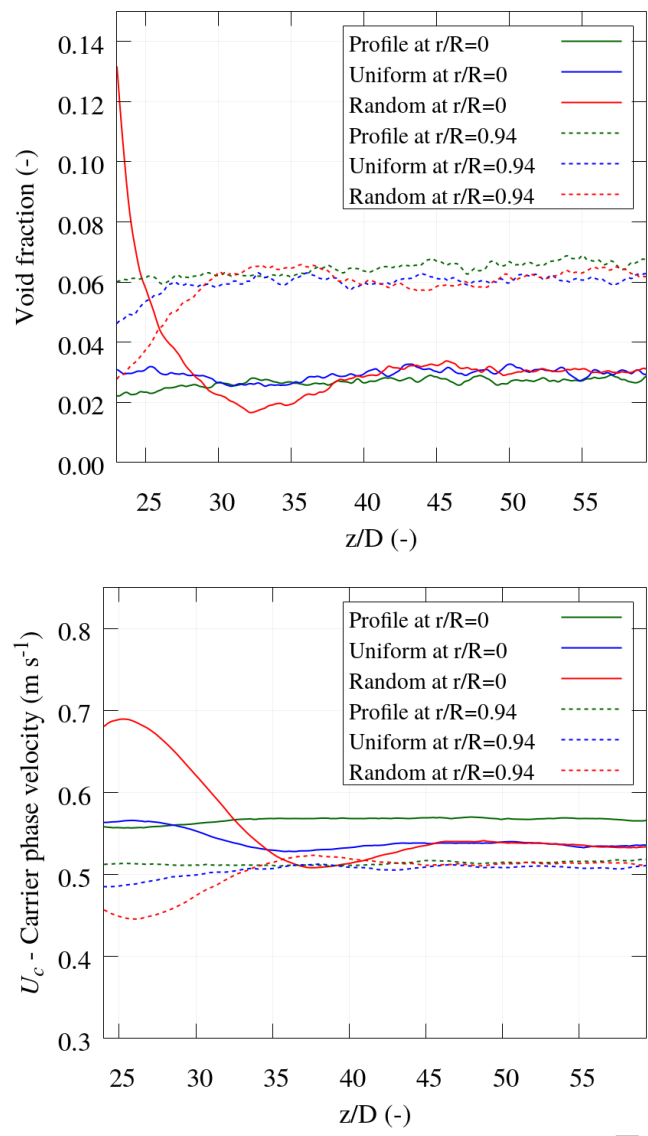

Figure 19: Axial evolution of void fraction and carrier phase velocity at several 883 radial locations according to the different seeding patterns analyzed.

same conditions of the experiments.

\section{Bubble size distribution and axial evolution}

According to the particle size, a disperse multiphase flow can be classified as monodisperse (uniform size) or polydisperse (nonuniform size) flow. In many simulations we can assume the ${ }^{889}$

bubble size is uniform either because the dispersion of the $\mathrm{BSD}_{890}{ }^{861}$ To assess and validate the model, we show first in Fig. 21 the is small enough, or because to assume monodispersity does not ${ }_{891}{ }^{86}{ }_{2 x i a l}$ evolution of the BSD, comparing the equivalent diameter compromise the quality of the results. But in general, flow char- ${ }_{892}{ }_{86} \mathrm{at} \mathrm{z} / \mathrm{D}=22.4$ and $\mathrm{z} / \mathrm{D}=98.7$.

acteristics depend on BSD polydisperse nature so it must be ${ }_{893}{ }^{864}$ The measured BSD shows the expected bubble size expanincluded for detailed and accurate simulations.

894865 sion with height. In the simulation, the BSD was extracted

In bubbly flows the bubble size is an important parameter to 89586 from the total bubbles in the system at a given height. Bubpredict the flow characteristics, and is needed to adequately de-896 ${ }_{86}$ ble size distribution at the two given heights are similar as the scribe the size distribution in space and time. To predict the $897{ }_{86}$ measured.

axial evolution of bubbly flow we must consider the pressure ${ }_{898}{ }_{869}$ The significant changes in volume and bubble size may inchanges along the pipe having a significant influence on bub-899 87 fluence on the bubble evolution along the pipe. For the sake of ble size and bubble volume. The axial evolution of the pres-900 87 argument, we examine in (see Fig. 22) the void fraction radial sure for the PW05003 scenario is shown in Fig. 20 to show this9o1 87profiles at the top measurement port for the following three scechange. The simulation was compared with the experiments to902 87 narios: $a$ ) an inlet uniform size with a mean diameter equivalent ensure that the pressure values are accurate to apply an expan-903 87to the mean of the distribution, $b$ ) an inlet uniform size that insion model.

being $p_{\mathrm{H}}$ the hydrostatic absolute pressure computed from

$$
p_{\mathrm{H}}=p_{\mathrm{atm}}+\rho_{\mathrm{c}} p+\alpha_{\mathrm{c}} \rho_{\mathrm{c}} g h,
$$
904 87cludes the bubble expansion and $c$ ) a polydisperse flow with the 

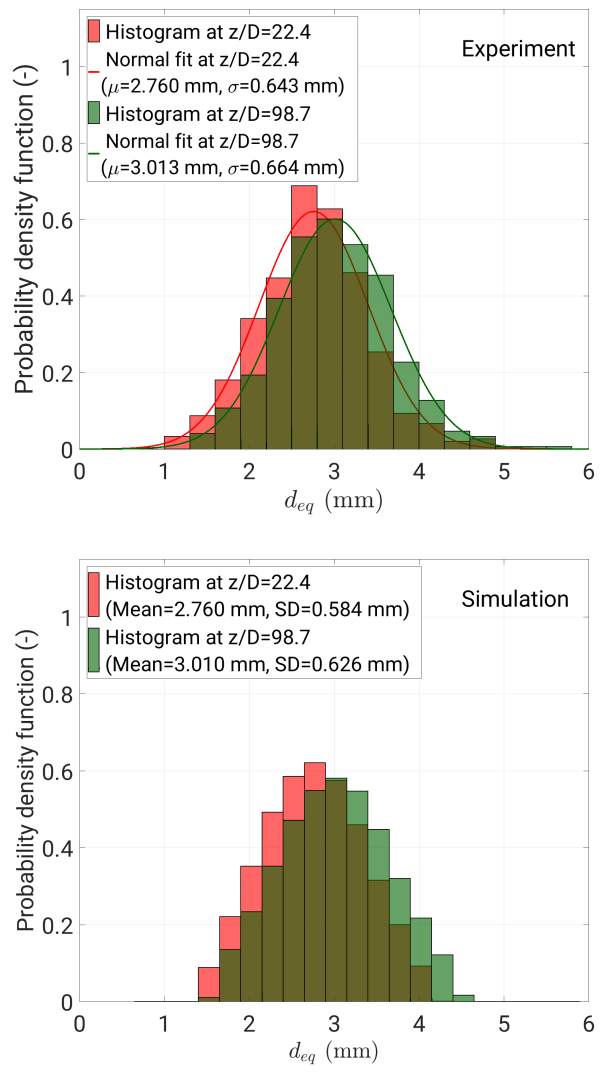

Figure 21: Bubble size distributions for experiments and simulations. Statistical parameters of the distribution are shown for the experiments and mean and standard deviation of the sample for the simulation.

95\% of the bubble sizes considered from the experimental size distribution at $\mathrm{z} / \mathrm{D}=22.4$, including in like manner, the bubble expansion..

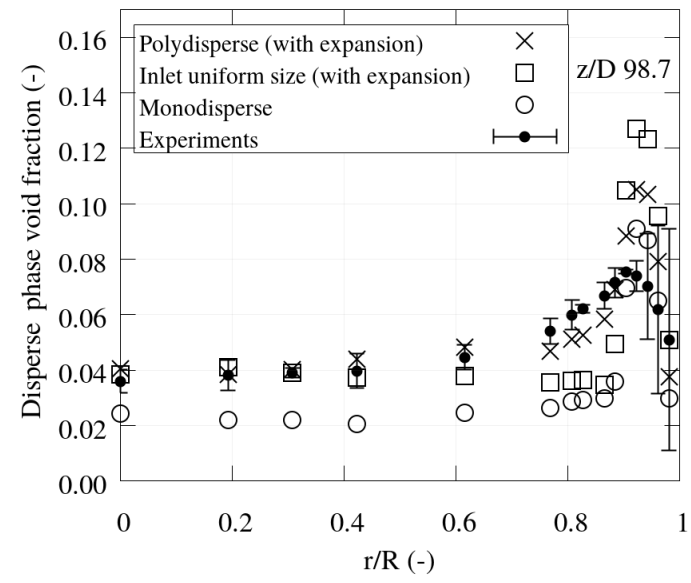

Figure 22: Disperse phase void fraction at $z / D=98.7$ for PW05003 comparing three cases: polydisperse with expansion ( $95 \%$ of the bubble sizes of the BSD), ${ }^{947}$ inlet uniform size with expansion (mean size of the BSD) and monodisperse 948 (mean size of the BSD).

Note that the monodisperse case underpredicts the void frac-os tion as shown in the figure as the bubble expansion can not be952 ${ }_{00 s}$ struction of the turbulence produced by the interfacial effects

$$
\begin{aligned}
\frac{\partial}{\partial t}\left(\alpha_{\mathrm{c}} \kappa_{\mathrm{c}}\right)+\nabla \cdot\left(\alpha_{\mathrm{c}} \mathbf{U}_{\mathrm{c}} \kappa_{\mathrm{c}}\right)=\nabla \cdot\left[\alpha_{\mathrm{c}}\left(v_{\mathrm{c}}+\frac{v_{t, \mathrm{c}}}{\sigma_{\kappa}}\right) \nabla \kappa_{c}\right] \\
+\alpha_{\mathrm{c}} \mathbf{R}_{\mathrm{c}}:\left(\nabla \mathbf{U}_{\mathrm{c}}\right)-\alpha_{\mathrm{c}} \epsilon_{\mathrm{c}} \\
-\mathbf{M}^{\mathrm{h}}\left(\mathbf{U}_{\mathrm{d}}-\mathbf{U}_{\mathrm{c}}\right)-\left(p_{\mathrm{d}}-p_{\mathrm{c}}\right) \frac{\delta \alpha_{d}}{\delta t}-\gamma \Gamma a_{I},
\end{aligned}
$$

where $v_{t, \mathrm{c}}$ is the eddy viscosity, $\varepsilon_{\mathrm{c}}$ is the turbulent dissipation rate, and $\Gamma$ is the rate of change of interfacial area.

The turbulent Reynolds stress is defined as Serizawa et al. (1975); Troshko and Hassan (2001a):

$$
\mathbf{R}_{\mathrm{c}}=v_{t, \mathrm{c}}\left(\nabla \mathbf{U}_{\mathrm{c}}+\left(\nabla \mathbf{U}_{\mathrm{c}}\right)^{\mathrm{T}}-\frac{2}{3} \mathbf{I}\left(\nabla \cdot \mathbf{U}_{\mathrm{c}}\right)\right)-\frac{2}{3} \mathbf{I} \kappa_{\mathrm{c}}
$$

The three last terms in the RHS of Eq. 28 are related to the interfacial effects on the turbulence kinetic energy. In particular the last two terms are included to be consistent with the expansion of the bubble described previously.

The turbulent dissipation rate equation used is based on the Kolmogorov's hypothesis (Pope, 2000). The production and dissipation rates of $\epsilon_{c}$ are considered proportional to the proaccording to Launder and Spalding (1974). Conversely, the de- considered to preserve the monodisperse constraint. The difference between the polydisperse and the inlet uniform size reveal significant discrepancies on the void fraction close to the wall. This can be attributed to differences in force balances, interactions with the wall and contributions of volume in space from different bubble sizes. In addition to the void fraction, the effect of the polydispersity on other variables (e.g. bubble frequency, interfacial area concentration, chord lengths or Sauter mean diameter) result more significant and may lead to a mistaken interpretation of the validation. Therefore, the flow must be considered as polydisperse for an accurate comparison, even if coalescence or breakup are negligible.

\section{Bubble dispersion and pseudoturbulence}

\subsection{Carrier phase turbulence model}

The bubbles motion produces random velocity fluctuations along their trajectories in the carrier phase. This can be considered as a pseudoturbulence according to van Wijngaarden (1998) and means an increase of turbulent velocity fluctuations because of the pass of bubbles. This produces an excess energy that needs to be considered in the turbulence models. A $\kappa-\varepsilon$ turbulence model for gas-liquid two-phase flow similar to Kataoka and Serizawa (1989); Morel (1997); Troshko and Hassan (2001a) is used in this work. According to these models the interfacial effects are considered explicitly in the transport equations for these turbulent variables. The total mixture turbulent kinetic energy is obtained by the summation of the $\kappa_{k}$ equation and considering the gas-phase turbulence negligible compared with the liquid-phase turbulence (Kataoka and Serizawa (1989)). The following equation is obtained for $\kappa$ : duction and dissipation rates of $\kappa_{\mathrm{c}}$ with a factor of $\omega_{\mathrm{c}}=\varepsilon_{\mathrm{c}} / \kappa_{\mathrm{c}}$ 
must be related to the characteristic time scale of the pseudoturbulence produced by the bubbles (Lopez de Bertodano, 1998; Troshko and Hassan, 2001a) with a dissipation frequency $\omega_{\mathrm{d}}$ that needs to be modeled. The turbulence dissipation rate equation results:

$$
\begin{aligned}
\frac{\partial}{\partial t}\left(\alpha_{\mathrm{c}} \varepsilon_{\mathrm{c}}\right)+ & \nabla \cdot\left(\alpha_{\mathrm{c}} \mathbf{U}_{\mathrm{c}} \varepsilon_{\mathrm{c}}\right)=\nabla \cdot\left[\alpha_{\mathrm{c}}\left(\nu_{\mathrm{c}}+\frac{v_{t, \mathrm{c}}}{\sigma_{\varepsilon}}\right) \nabla \varepsilon_{c}\right] \\
& +\omega_{\mathrm{c}}\left(C_{\varepsilon, 1} \alpha_{\mathrm{c}} \mathbf{R}_{\mathrm{c}}:\left(\nabla \mathbf{U}_{\mathrm{c}}\right)-C_{\varepsilon, 2} \alpha_{\mathrm{c}} \varepsilon_{\mathrm{c}}\right) \\
& -\omega_{\mathrm{d}}\left(\mathbf{M}^{\mathrm{h}}\left(\mathbf{U}_{\mathrm{d}}-\mathbf{U}_{\mathrm{c}}\right)+\left(p_{\mathrm{d}}-p_{\mathrm{c}}\right) \frac{\delta \alpha_{d}}{\delta t}+\gamma \Gamma a_{i}\right),
\end{aligned}
$$

where $C_{\varepsilon, 1}, C_{\varepsilon, 2}$ and $\sigma_{\varepsilon}$ are defined as in Launder and Spalding (1974); Troshko and Hassan (2001a). The dissipation frequency of the production terms produced by the bubbles, $\omega_{\mathrm{d}}$, was modeled in this work as suggested by Morel (1997) (see Eq. 31). This is based on the relation of proportionality between interfacial turbulence production and dissipation of Elghobashi and Abou-Arab (1983) and defining the characteristic time $\tau$ with the diameter of the bubble as the length scale ${ }^{1012}$ (Morel, 1997; Yao and Morel, 2004). Note that in our simula- ${ }^{1013}$ tions the characteristic time can be defined, for accuracy, with the actual bubble diameter and not the Sauter mean diameter as in the mentioned works.

$$
\omega_{\mathrm{d}}=\frac{C_{\varepsilon, 3}}{\tau}=\frac{C_{\varepsilon, 3}}{\left(d_{b}^{2} / \varepsilon_{\mathrm{c}}\right)^{\frac{1}{3}}}
$$

The constant $C_{\varepsilon 3}$ was set to 1.0 in Yao and Morel (2004) for 1016 adiabatic scenarios similar to the ones studied in this paper.

Finally, the law of the wall of Troshko and Hassan (2001a,b) for two-phase turbulent boundary layers was implemented in the solver to obtain a more accurate description of the turbulence near the wall.

\subsection{Bubble dispersion model}

On the other hand, when using a RANS turbulence model ${ }_{3025}$ the average velocity $\mathbf{U}_{\mathrm{c}}$ is solved. To calculate adequately the $e_{026}$ forces including the liquid-phase turbulence effect, the instan ${ }_{\text {To2 }}$ taneous carrier phase velocity seen by the bubbles is modeled. The fluctuating velocity component $\mathbf{u}_{\mathrm{c}}^{\prime}$ has been modeled in the past using Discrete Random Walk (DRW) (Buwa et al. (2006); Gosman and Loannides (1983)) and continuous random walk (CRW) (Thomson (1987)) stochastic models. The latter solves the Langevin equation and provides a more accurate solution of the particle path than DRW. It should be noted that Large Eddy Simulation (LES) could be used, in principle, to model the turbulence and compute the velocity fluctuations. To apply these models implies notable grid restrictions that are inconsistent with the unresolved CFD-DEM approach for the conditions of this work. Then, we use the two-equation turbulence for simplicity and to reduce the computational costs.

The velocity fluctuations can be obtained directly for homogeneous turbulence through a Markov chain based on the Langevin equation. In wall-bounded flows, the turbulence is expected to be inhomogeneous. Wilson et al. (1981); Iliopoulos and Hanratty (1999) proposed a normalized Langevin equation to consider the inhomogeneous turbulence. In addition, a drift correction was included to consider in the Markov chain the inhomogeneity and to avoid non-physical diffusion (MacInnes and Bracco, 1992; Bocksell and Loth, 2006). For instance, it provides that tracer particles will follow streamlines on average (Dehbi, 2008), but particles with a large Stokes number will have little influence from the fluid motion.

The normalized Langevin equation for isotropic inhomogeneous turbulence can be expressed as Dehbi (2008) to get the $\mathbf{u}_{\mathrm{c}}^{\prime}$ for each bubble during the time. In this work the drift correction term is considered noninertial for bubbles, and the equation results:

$$
\mathrm{d}\left(\frac{\mathbf{u}_{\mathrm{c}}^{\prime}}{u_{\mathrm{rms}}}\right)=-\left(\frac{\mathbf{u}_{\mathrm{c}}^{\prime}}{u_{\mathrm{rms}}}\right) \frac{\mathrm{d} t}{\tau}+\sqrt{\frac{2}{\tau}} \mathrm{d} \xi+\frac{1}{3 u_{\mathrm{rms}}} \nabla \kappa_{\mathrm{c}} \mathrm{d} t,
$$

where $\mathrm{d} \xi$ is a succession of uncorrelated Gaussian random 1010 numbers with zero mean and variance $\mathrm{d} t$ for each direction.

From the definition of mean kinetic energy of the turbulence and for isotropic turbulence (Pope, 2000):

$$
u_{\mathrm{rms}}=u_{\mathrm{x}, \mathrm{rms}}=u_{\mathrm{y}, \mathrm{rms}}=u_{\mathrm{z}, \mathrm{rms}}=\sqrt{\frac{2}{3} \kappa_{\mathrm{c}}}
$$

\subsection{Validation of the turbulence and dispersion models and in- fluence on the results}

Finally, we analyze the effects of both, CRW and two-phase flow turbulence model on the liquid instantaneous velocity and on the disperse phase characteristics.

Figure 23 shows the instantaneous liquid velocity $\mathbf{u}_{\mathrm{c}}$ obtained with the LDA at $\mathrm{z} / \mathrm{D}=98.7$ and $\mathrm{r} / \mathrm{R}=0$ and the computational results. In the simulation, the bubbles passing by a location coincident with the LDA measurement location are selected to show $\mathbf{u}_{\mathrm{c}}$.

The bubble velocity distribution was analyzed at the same position $(\mathrm{z} / \mathrm{D}=98.7$ and $\mathrm{r} / \mathrm{R}=0)$ in Fig. 24. When no $\mathrm{CRW}$ is included (top figure), the bubble velocity distribution is dominated by the drag correlation. Then, the different velocities are explained mainly by the different bubble sizes. This results in a clear underprediction in the bubble velocity fluctuations. When the CRW is included (middle figure), the bubble velocity distribution is clearly wider and looks similar to the experimental one (bottom figure).

The turbulence effects on the void fraction are analyzed. We consider different combinations neglecting the CRW and the gsturbulence produced by the bubbles (BPT). When the CRW is ssnot considered we assume $\mathbf{u}_{\mathrm{c}}=\mathbf{U}_{\mathrm{c}}$, and to neglect the BPT we ${ }_{98}$ cancel the last three terms in Eq. 28 and Eq. 30. These tests g9are shown in Fig. 25. The CRW model has a major impact ${ }_{99}$ for the time-averaged void fraction profiles, noting a clear ef${ }_{99}$ fect near the wall. When the liquid velocity fluctuations were ${ }_{99}$ not considered, the void fraction peak was further accentuated g9and minimum values were found close to this area. In contrast, 99: gothe bubbles effects, a more accurate profile can be obtained. 

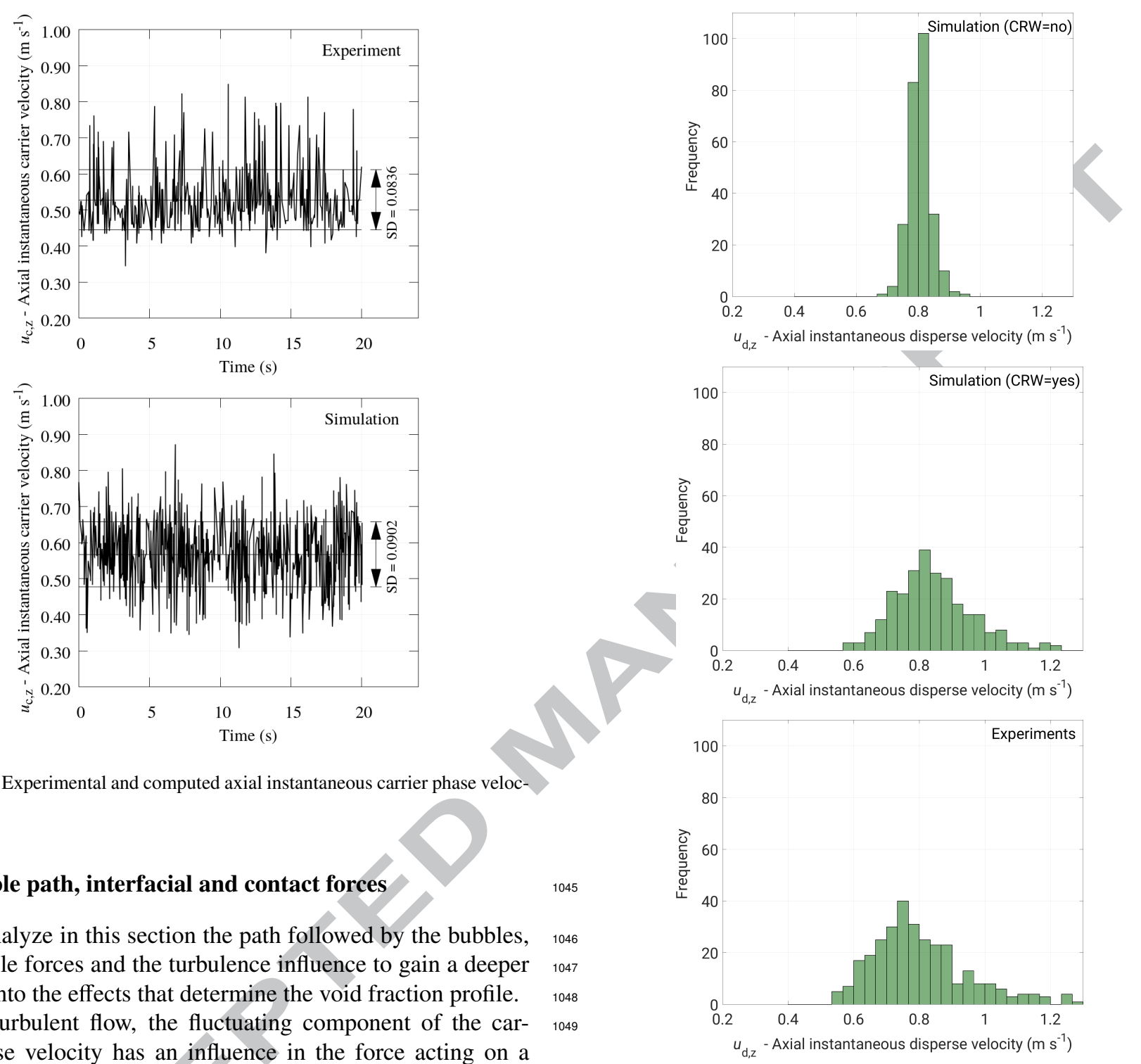

Figure 23: Experimental and computed axial instantaneous carrier phase velocity.

\section{Bubble path, interfacial and contact forces}

We analyze in this section the path followed by the bubbles, the bubble forces and the turbulence influence to gain a deeper insight into the effects that determine the void fraction profile.

In a turbulent flow, the fluctuating component of the carrier phase velocity has an influence in the force acting on a bubble. Essentially they are captured in turbulent eddies and moved with it. Usually, the turbulence effect on the interfacial forces are neglected in TFM or are only considered in the drag through the turbulent dispersion force (Lopez de Bertodano, 1992; Burns et al., 2004). The influence of the turbulence on the interfacial forces was evaluated by Behzadi et al. (2001) for mixing layer and sudden expansion scenarios concluding that the turbulent effects on lift and virtual mass forces are negligible for these cases. However, in the literature, there are no many investigations showing its influence in other systems. Indeed, when the lateral forces are predominant as in the case of wall-bounded systems we may expect an important influence on the lift and wall interaction turbulent effects. With the solver presented in this work the turbulence effects are considered directly through the instantaneous velocities used to calculate the forces.

In the pipe system simulated we follow the path of bubbles of similar diameter $(2.7-2.8 \mathrm{~mm})$ and seeded at the inlet in the vicinity of the wall ( $\mathrm{r} / \mathrm{R}>0.9)$. The results are shown in Fig. 26.

The figure represents the normalized axial distance against the normalized distance to wall of the bubble center. The figure
Figure 24: Comparison between experiments and simulations of the probability density function of the disperse phase velocity at $\mathrm{z} / \mathrm{D}=98.7$ and $\mathrm{r} / \mathrm{R}=0$. Simulations performed with and without CRW.

on the left side shows the paths followed when CRW is not included. In this case, the bubbles studied tend to an equilibrium position that results mainly from a force balance between lift and contact bubble-wall. In contrast, when the CRW is used (right figure), the fluctuation effects are clear. Some bubbles leave the equilibrium state presumably because of the dispersion effects. Furthermore, because of an increase in the number and kinetic energy of the collisions with the wall, it leaves the bubble beyond the range of influence of the lift force. This produces a temporal migration of some bubbles close to the center. This is more significant as the bubble increase its size with the height. We can compare the presence of bubbles in the range of $\mathrm{r} / \mathrm{R}$ between 0.75 to 0.9 for both cases and the corresponding void fraction shown before in Fig. 25, noting consistent results. 1070 In addition, we select bubbles from small to large diameters ${ }_{107}$ present in the simulation. The normalized distance is plot-
1072 1073 1074 1075 1076 1077 1078 1079 1080 1081 1082 1083 1084 1085 1086 1087 

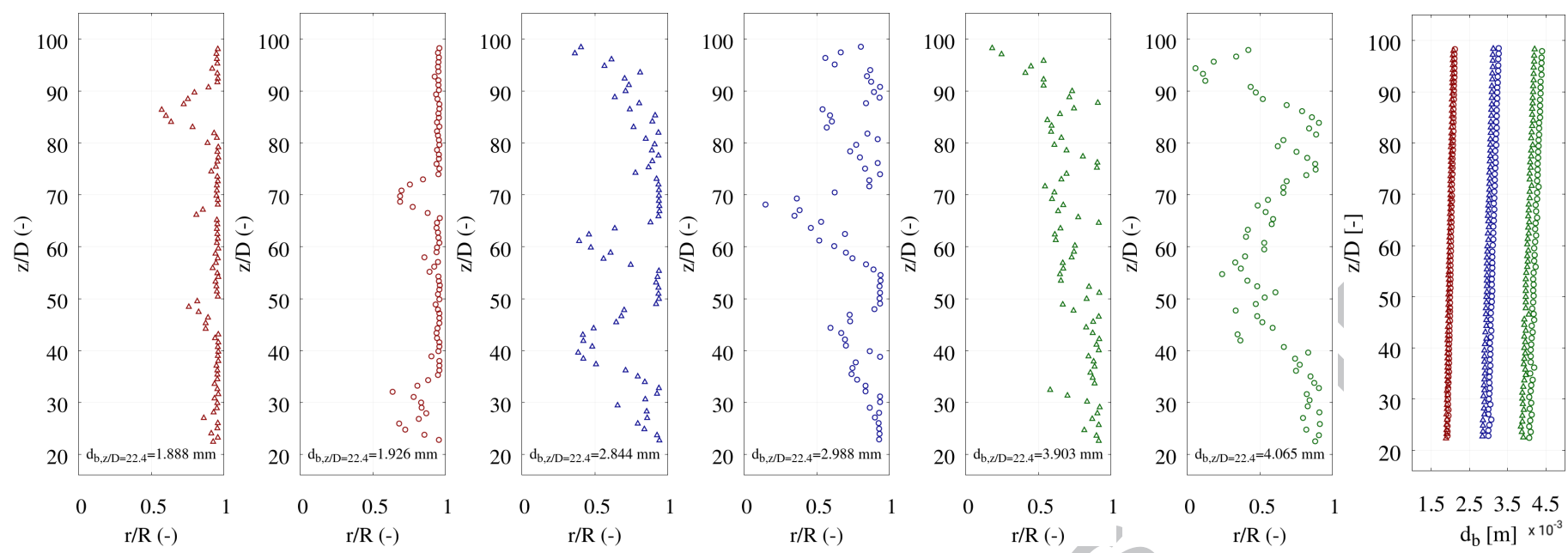

Figure 27: Distance to wall along the axial distance.

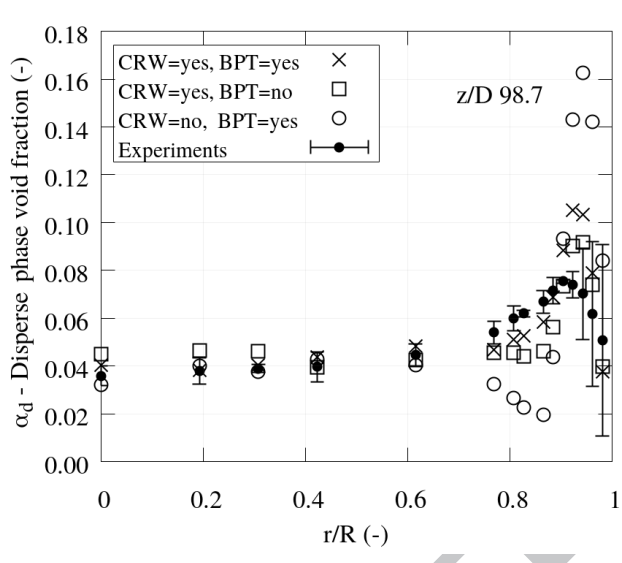

Figure 25: Disperse phase void fraction at $\mathrm{z} / \mathrm{D}=98.7$ for $\mathrm{PW} 05003$ comparing the influence of the turbulence effects with CRW and BPT.
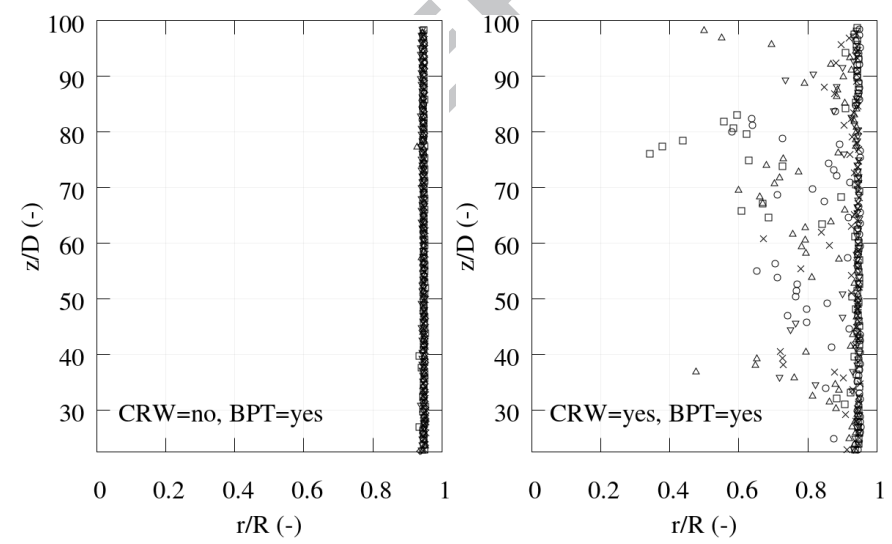

Figure 26: Distance to wall along the axial distance of 5 randomly selected bubbles of size 2.7 to $2.8 \mathrm{~mm}$ and seeded at $\mathrm{r} / \mathrm{R}>0.9$ for simulations with and without the CRW stochastic model. Each symbol represents the distance to wall of each bubble. travel far from the wall in the pipe. As a consequence, they con- 1091 tribute on the void fraction more further from the wall without 1092 this meaning a lateral migration deriving from a negative lift ${ }_{1093}$ force coefficient. Furthermore, the axial bubble size increase 1094 makes some bubbles more favorable to bounce during their rising.

Next, we study the behavior of the bubbles in the radial direction. At each time step, we analyze velocity and forces of bubbles with centroid coordinates at $\mathrm{x} \geq 0,-0.5 \leq \mathrm{y} \leq 0.5$ and $\mathrm{z} \pm 50$ $\mathrm{mm}$. The radial bubble velocity at the bubble centroid is shown in Fig.28.

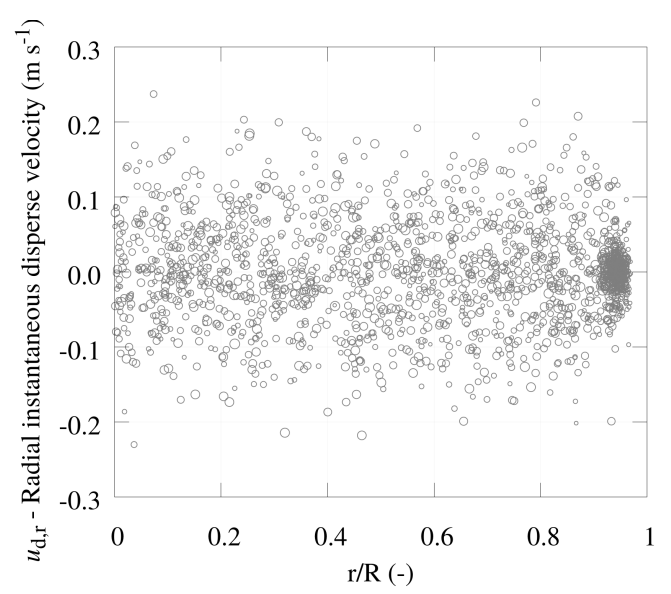

Figure 28: Computational results of radial bubble velocity at the bubble centroid position.

Positive and negative values occur because of the turbulence effects. Close to the wall it is also produced by the bounces of the bubbles as they approach to the wall bouncing back to the main flow. In this region, an accumulation of bubbles with a velocity near to zero is appreciated because of the bubble-wall interaction.

ted against the distance to wall for bubbles of different size 1081088 The lateral forces are responsible for the void fraction radial (Fig. 27). In the figure, one can appreciate how smaller bub +109108 profile and the path of the bubbles. We analyze in Fig. 29 the bles tend to rise close to the wall. In contrast, bigger bubbles ${ }_{110} 109$ radial component of lift and wall lubrication force at the bubble 
centroid. These results show that the wall lubrication force is negligible, for this case, in comparison to the lift force. Close to the wall positive values of lift force are found as expected, with different contributions depending on the bubble size.

Negative values of the lift forces are also appreciated although only positive values of the lift coefficients are given. This is produced by the existence of local velocity gradients in the opposite direction to the average gradient.
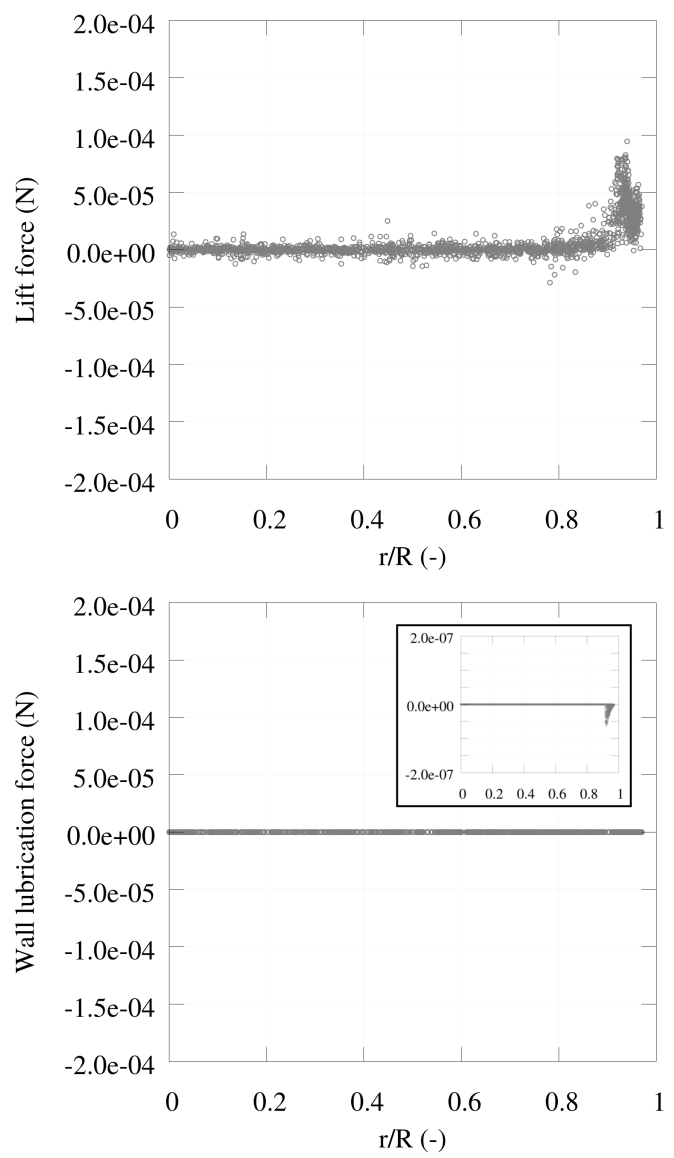

Figure 29: Computational results of radial component of lift and wall lubrication forces at the bubble centroid position.

\section{Conclusions}

We developed a new CFD-DEM solver using the OpenFOAM ${ }^{\circledR}$ library. To exclude complex phenomena such as bubble breakup and coalescence, the present study was limited to a specific bubbly flow condition in vertical pipes. Then, the flow dynamics is expected to be governed by the bubble forces and the coupling between phases. Thus, the solver was configured to include these phenomena.

The solver includes the coupling between phases, the required interfacial forces, a two-equation turbulence model for two-phase flow and the bubble-bubble and bubble-wall interaction with a soft-sphere model. Several contributions were required, in addition, to develop the presented CFD-DEM solver.

The first consisted of a new subelement method to provide stability and precision on the exchange of momentum and volume fraction, basing on equivolumetric divisions of the spheres.

Once the coupling between the phases was performed accurately, we provided a correct inlet condition developing an algorithm to seed the bubbles as in the experiment. The new proposed algorithm allows defining the locations, velocities and sizes of the bubbles to match any required inhomogeneous bubble size distribution.

Furthermore, the changes in size and volume of the bubbles produced by the pressure variations were investigated experimentally. A simplified model was integrated in the bubble tracking through the Young-Laplace equation and the ideal gas law. The results showed that this model were required to get accurate result. In addition, a polydisperse and a uniform inlet case were compared to demonstrate that considering the dynamics of the different sizes, and bubble expansion of the population led to better results.

With respect to the turbulence modeling, a two-phase flow $\kappa-\varepsilon$ model was used as the CFD-DEM formulation limits the grid refinement in the near-wall region. To include the turbulent dispersion, a Continuous Random Walk stochastic model was used to calculate the instantaneous liquid velocity seen by every bubble. Bubble pseudoturbulence was considered in the $\kappa-\varepsilon$ including the effects of the bubble expansion. The instantaneous liquid velocity and the probability density function of the bubble velocity were properly captured using these models comparing with the experiments. The relevance of the turbulent effects was clearly shown in the void fraction profile and bubble paths. Noting that the dispersion was accounted for in this work without the need of a turbulent dispersion force and any tuning coefficient.

The path of the bubbles was analyzed focusing the attention to the distance to the wall at different axial positions. The bubble dynamics are determined mainly by the lift force interaction, the bubble-wall contact forces and the turbulence effects. According to the results, when the CRW was used, the bubbles close to the wall leave the equilibrium position and they travel through distance far from the wall. The study of the path for different bubble sizes reveals that the small bubbles tend to rise close to the wall, while bigger bubbles spend more time away from the wall. Finally, the lift force and wall lubrication force were shown indicating that the latter had a negligible influence for this case.

In summary, this work allowed simulating bubbly flow systems with bubbles represented as discrete elements to analyze the two-phase flow characteristics in different pipes. Let us note that the proposed solver can be further extended to other flow regimes including more complex phenomenology as nonsphericity of bubbles, near-wall modeling, and bubble breakup or coalescence. This study can be used for a direct comparison of simulations in pipes with two-fluid model using the same models as with the CFD-DEM approach. This could be useful 113to interpret the effects of the assumptions made in the two-fluid ${ }_{113}$ model. 


\section{Acknowledgments}

The authors sincerely thank the "Plan Nacional de I+D+i" for funding the project MODEXFLAT ENE2013-48565-C2-1$\mathrm{P}$ and ENE2013-48565-C2-2-P.

\section{Appendix A. Mesh sensitivity analysis}

In this section, the influence of the mesh density on the results is examined with three configurations: coarser, medium and finer. A pipe of $1 \mathrm{~m}$ length and $52 \mathrm{~mm}$ diameter are used. The medium mesh corresponds to the same O-grid as the one used in the whole paper shown previously in Fig. 10. Fig. A.30 shows the configuration of coarser and finer meshes. The parameters defining the $\mathrm{O}$-grid are indicated in this figure. The number of cells in the radial, $n_{r}$, and tangential, $n_{t}$, direction in the $\mathrm{O}$-grid are modified to obtain different mesh densities. The grid spacing in the axial direction, $n_{a}$, is uniform and fixed to 1.5 times the maximum bubble diameter to satisfy the grid restrictions of the CFD-DEM solver for all three meshes. The first node close to the wall is placed to a defined distance in order to keep the nondimensional wall distance, $y^{+}$, around 11.6

The partition of the bubbles is performed as described in Section 4 . The simulation with the three meshes were run with the same value of $\delta r$ obtained from the analysis performed in that section for the medium mesh. For the coarser and finer meshes we also verified that the maximum ratio of total volume occupied by the bubbles and volume cell obtained at each time step was far below 1 .

Table A.4 shows the main parameters of the three meshes and the wall-clock time needed for the simulation.

Table A.4: Flow conditions and studied variables used in this work.

\begin{tabular}{llllll}
\hline Mesh & $n_{r}$ & $n_{t}$ & $n_{a}$ & No. of cells & $\begin{array}{l}\text { Wall-clock time } \\
(\mathrm{s})\end{array}$ \\
\hline Coarser & 6 & 7 & 166 & 33864 & 242532 \\
Medium & 8 & 8 & 166 & 53120 & 323242 \\
Finer & 10 & 9 & 166 & 76360 & 470103 \\
\hline
\end{tabular}

The results of the simulations are shown in Fig. A.31. Carrier phase velocity and turbulence intensity are selected to analyze the influence of the mesh density on the carrier phase. Void fraction is shown to determine its influence on the disperse phase.

The results show that there are no drastic differences among the different mesh densities analyzed. Slight differences are appreciated at the peak of the void fraction profile comparing the coarser configuration with the medium and finer ones. For the carrier phase velocity and turbulence intensity the differences are appreciated in the radial profile. Medium and coarser meshes present almost similar results for the three variables. As the medium configuration takes less computational time, it was chosen to perform the simulations presented in the paper.

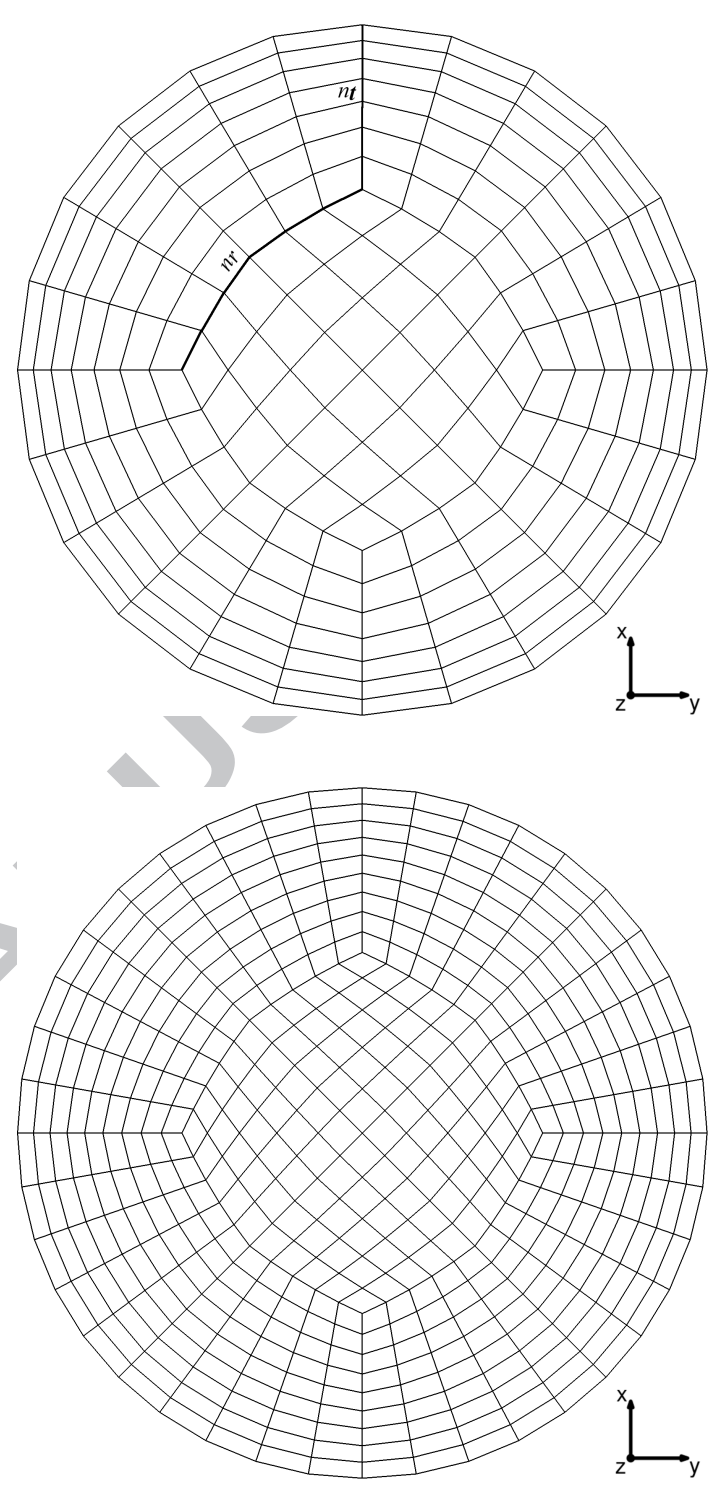

Figure A.30: Cross-section mesh view for coarser (up) and finer (down) configurations.

\section{References}

Alajbegovic, A., Drew, D. A., Lahey, R. T., aug 1999. An analysis of phase distribution and turbulence in dispersed particle/liquid flows. Chemical Engineering Communications 174 (1), 85-133.

Alam, M., Luding, S., 2003. Rheology of bidisperse granular mixtures via eventdriven simulations. Journal of Fluid Mechanics 476, 69-103.

Antal, S. P., Lahey Jr, R. T., Flaherty, J. E., 1991. Analysis of phase distribution in fully developed laminar bubbly two-phase flow. International Journal of Multiphase Flow 17 (5), 635-652.

Auton, T. R., Hunt, J. C. R., Prud'Homme, M., 1988. The force exerted on a body in inviscid unsteady non-uniform rotational flow. J. Fluid Mech. 197, 241-257.

Barrau, E., Rivière, N., Poupot, C., Cartellier, A., mar 1999. Single and double optical probes in air-water two-phase flows: real time signal processing and sensor performance. International Journal of Multiphase Flow 25 (2), 229256.

Behzadi, A., Issa, R. I., Rusche, H., 2001. Effects of turbulence on inter-phase forces in dispersed flow. In: ICMF'2001, 4th Int. Conf. Multiphase Flow. pp. 1-12.

Besagni, G., Brazzale, P., Fiocca, A., Inzoli, F., 2016. Estimation of bubble size 

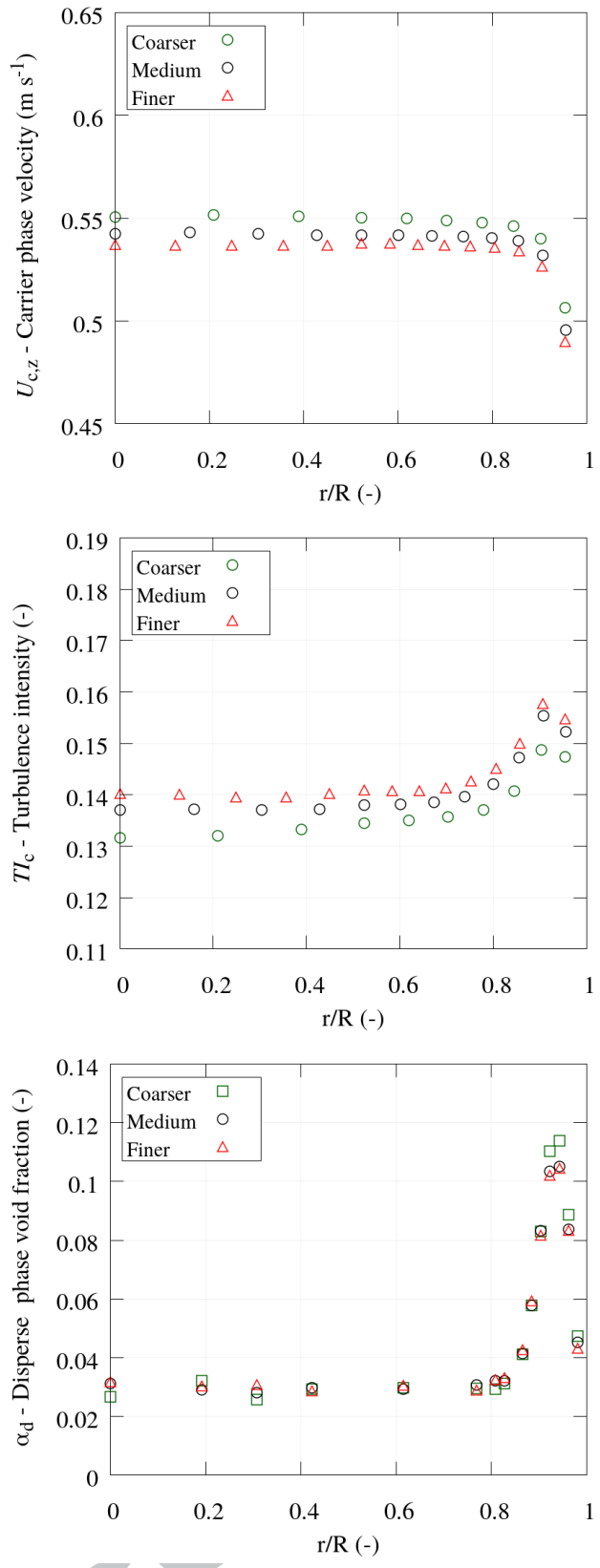

Figure A.31: Results of the mesh sensitivity analysis.

distributions and shapes in two-phase bubble column using image analysis and optical probes. Flow Measurement and Instrumentation 52, 190-207.

Besagni, G., Inzoli, F., jun 2016. Bubble size distributions and shapes in annular gap bubble column. Experimental Thermal and Fluid Science 74, 27-48.

Besagni, G., Inzoli, F., Ziegenhein, T., Lucas, D., mar 2017. Computational Fluid-Dynamic modeling of the pseudo-homogeneous flow regime in largescale bubble columns. Chemical Engineering Science 160, 144-160.

Bocksell, T. L., Loth, E., 2006. Stochastic modeling of particle diffusion in a turbulent boundary layer. International Journal of Multiphase Flow 32, 1234-1253.

Burns, A. D., Frank, T., Hamill, I., Shi, J.-M., 2004. The Favre averaged drag model for turbulent dispersion in Eulerian multi-phase flows. In: 5th international conference on multiphase flow, ICMF. Vol. 4. pp. 1-17.

Buwa, V. V., Deo, D. S., Ranade, V. V., 2006. EulerianLagrangian simulations of unsteady gasliquid flows in bubble columns. International Journal of Multiphase Flow 32 (7), 864-885.

Cai, Z., Bao, Y., Gao, Z., dec 2010. Hydrodynamic Behavior of a Single Bubble
Rising in Viscous Liquids. Chinese Journal of Chemical Engineering 18 (6), 923-930.

Crowe, C. T., Sharma, M. P., Stock, D. E., jun 1977. The Particle-Source-In Cell (PSI-CELL) Model for Gas-Droplet Flows. Journal of Fluids Engineering 99 (2), 325-332.

Crowe, C. T. C. T., 2006. Multiphase flow handbook. CRC.

Cundall, P. A., Strack, O. D. L., 1979. A discrete numerical model for granular assemblies. Géotechnique 29 (1), 47-65.

de Vries, A. W. G., feb 2001. Path and wake of a Rising Bubble. Ph.D. thesis, University of Twente, Enschede.

Deen, N. G., van Sint Annaland, M., Kuipers, J. A. M., 2004. Multi-scale modeling of dispersed gasliquid two-phase flow. Chemical Engineering Science 59 (89), 1853-1861.

Dehbi, A., 2008. Turbulent particle dispersion in arbitrary wall-bounded geometries: A coupled CFD-Langevin-equation based approach. International Journal of Multiphase Flow 34, 819-828.

Delnoij, E., Lammers, F. A., Kuipers, J. A. M., van Swaaij, W. P. M., 1997. Dynamic simulation of dispersed gas-liquid two-phase flow using a discrete bubble model. Chemical Engineering Science 52 (9), 1429-1458.

Drew, D. A., Lahey, R. T., 1987. The virtual mass and lift force on a sphere in rotating inviscid flow. Int. J. Multiphase Flow 13 (1), 113-121.

Dukowicz, J. K., 1980. A particle-fluid numerical model for liquid sprays. Journal of Computational Physics 35 (2), 229-253.

E. Shams, Finn, J., Apte, S. V., 2010. A Numerical Scheme for Euler-Lagrange Simulation of Bubbly Flows in Complex Systems. International Journal for Numerical Methods in Fluids.

Ekambara, K., Sanders, R. S., Nandakumar, K., Masliyah, J. H., 2008. CFD simulation of bubbly two-phase flow in horizontal pipes. Chemical Engineering Journal 144 (2), 277-288.

Elghobashi, S. E., Abou-Arab, T. W., 1983. A two-equation turbulence model for two-phase flows. Physics of Fluids 26 (4), 931-938.

Essa, M. A. A. E. A., 2012. Coupled Lagrange-Euler model for simulation of bubbly flow in vertical pipes considering turbulent 3D random walks models and bubbles interaction effects. Ph.D. thesis, Universitat Polit\{̇̀\}cnica de Val\{è\}ncia.

Fox, R. O., 2012. Large Eddy Simulation Tools for Multiphase Flows. Annual Review of Fluid Mechanics 44 (1), 47-76.

Gosman, A. D., Loannides, E., 1983. Aspects of computer simulation of liquidfueled combustors. Journal of Energy 7 (6), 482-490.

Guédon, G. R., Besagni, G., Inzoli, F., apr 2017. Prediction of gasliquid flow in an annular gap bubble column using a bi-dispersed Eulerian model. Chemical Engineering Science, 138-150.

Gui, N., Fan, J. R., Luo, K., 2008. DEM-LES study of 3-D bubbling fluidized bed with immersed tubes. Chemical Engineering Science 63 (14), 3654 3663.

Gunsing, M., jan 2004. Modelling Bubbly flows using volume of fluid, front tracking and discrete bubble models. Ph.D. thesis, University of Twente.

Gupta, A., Roy, S., 2013. Euler-Euler simulation of bubbly flow in a rectangular bubble column: Experimental validation with Radioactive Particle Tracking. Chemical Engineering Journal 225, 818-836.

Hilton, J. E., Mason, L. R., Cleary, P. W., 2010. Dynamics of gassolid fluidised beds with non-spherical particle geometry. Chemical Engineering Science 65 (5), 1584-1596.

Hosokawa, S., Tomiyama, A., 2009. Multi-fluid simulation of turbulent bubbly pipe flows. Chem. Eng. Sci. 64 (24), 5308-5318.

Iliopoulos, I., Hanratty, T. J., aug 1999. Turbulent dispersion in a nonhomogeneous field. Journal of Fluid Mechanics 392, 45-71.

Ishii, M., Hibiki, T., 2006. Thermo-fluid Dynamics of Two-Phase Flow. Smart Energy Systems. Springer.

Issa, R. I., 1986. Solution of the implicitly discretised fluid flow equations by operator-splitting. J. Comput. Phys. 62 (1), 40-65.

Jasak, H., 1996. Error analysis and estimation for finite volume method with applications to fluid flow. Ph.D. thesis, Imperial College London, University of London.

Kataoka, I., Serizawa, A., 1989. Basic equations of turbulence in gas-liquid two-phase flow. International Journal of Multiphase Flow 15 (5), 843-855.

Kazakis, N. A., Mouza, A. A., Paras, S. V., 2008. Experimental study of bubble formation at metal porous spargers: Effect of liquid properties and sparger characteristics on the initial bubble size distribution. Chemical Engineering Journal 137 (2), 265-281.

Kloss, C., Goniva, C., Hager, A., Amberger, S., Pirker, S., 2012. Models, al- 
gorithms and validation for opensource DEM and CFD-DEM. Progress in Computational Fluid Dynamics, An International Journal 12 (2/3), 140.

Krepper, E., Lucas, D., Prasser, H.-M., 2005. On the modelling of bubbly flow in vertical pipes. Nucl. Eng. Des. 235 (5), 597-611.

Kuang, S. B., Chu, K. W., Yu, A. B., Zou, Z. S., Feng, Y. Q., 2008. Computational Investigation of Horizontal Slug Flow in Pneumatic Conveying. Industrial \& Engineering Chemistry Research 47 (2), 470-480.

Laakkonen, M., Moilanen, P., Alopaeus, V., Aittamaa, J., 2007. Modelling local bubble size distributions in agitated vessels. Chemical Engineering Science 62 (3), 721-740.

Lage, P. L. C., Espósito, R. O., 1999. Experimental determination of bubble size distributions in bubble columns: prediction of mean bubble diameter and gas hold up. Powder Technology 101 (2), 142-150.

Lamb, H., 1895. Hydrodynamics. University Press.

Launder, B. E., Spalding, D. B., 1974. The numerical computation of turbulent flows. Comput. Method. Appl. M. 3 (2), 269-289.

Legendre, D., Magnaudet, J., aug 1998. The lift force on a spherical bubble in a viscous linear shear flow. Journal of Fluid Mechanics 368, S0022112098001621.

Legendre, D., Zenit, R., Velez-Cordero, J. R., apr 2012. On the deformation of gas bubbles in liquids. Physics of Fluids 24 (4), 043303

Lim, K. S., Agarwal, P. K., O'neill, B. K., 1990. Measurement and modelling of bubble parameters in a two-dimensional gas-fluidized bed using image analysis. Powder Technology 60 (2), 159-171.

Link, J. M., Cuypers, L. A., Deen, N. G., Kuipers, J. A. M., 2005. Flow regimes in a spoutfluid bed: A combined experimental and simulation study. Chemical Engineering Science 60 (13), 3425-3442

Lopez de Bertodano, M., 1992. Turbulent bubbly two-phase flow in a triangular duct. Ph.D. thesis, Rensselaer Polytechnic Institute.

Lopez de Bertodano, M., 1998. Two fluid model for two-phase turbulent jets. Nucl. Eng. Des. 179 (1), 65-74.

MacInnes, J. M., Bracco, F. V., 1992. Stochastic particle dispersion modeling and the tracerparticle limit. Physics of Fluids A: Fluid Dynamics 4 (12), 2809-2824

Macpherson, G., Reese, J., may 2008. Molecular Dynamics in Arbitrary Geometries: Parallel Evaluation of Pair Forces. Molecular Simulation 34 (01), $97-115$.

Macpherson, G. B., Nordin, N., Weller, H. G., 2009. Particle tracking in unstructured, arbitrary polyhedral meshes for use in CFD and molecular dynamics. Communications in Numerical Methods in Engineering 25 (3), 263 273.

Magnaudet, J., Eames, I., jan 2000. The Motion of High-Reynolds-Number Bubbles in Inhomogeneous Flows. Annual Review of Fluid Mechanics 32 (1), 659-708

Matuttis, H. G., Luding, S., Herrmann, H. J., 2000. Discrete element simulations of dense packings and heaps made of spherical and non-spherical particles. Powder Technology 109 (13), 278-292.

Monrós-Andreu, G., Martínez-Cuenca, R., Torró, S., Chiva, S., 2017. Local parameters of airwater two-phase flow at a vertical T-junction. Nuclear Engineering and Design 312, 303-316.

Monrós-Andreu, G., S. Chiva, R. Martínez-Cuenca, S. Torró, J. E. Juliá, L. Hernández, R. Mondragón, 2013. Water temperature effect on upward airwater flow in a vertical pipe: Local measurements database using foursensor conductivity probes and LDA. EPJ Web Conf. 45, 1105.

Morel, C., 1997. Mod\{é\}lisation multidimensionnelle des \{é\}coulements diphasiques gaz - liquide: application \{à\} la simulation des \{é\}coulements $\{a ̀\}$ bulles ascendants en conduite verticale. Ph.D. thesis, Ecole centrale de Paris.

Norouzi, H. R., Zarghami, R., Mostoufi, N., 2016a. New hybrid CPU-GPU solver for CFD-DEM simulation of fluidized beds. Powder Technology, - .

Norouzi, H. R., Zarghami, R., SotudehGharebagh, R., Mostoufi, N., sep 2016 b. CFD-DEM Formulation and Coupling. In: Coupled CFD-DEM Modeling. John Wiley \& Sons, Ltd, Chichester, UK, pp. 257-340.

Norouzi, H. R., Zarghami, R., SotudehGharebagh, R., Mostoufi, N., sep $2016 \mathrm{c}$. DEM Implementation. In: Coupled CFD-DEM Modeling. John Wiley \& Sons, Ltd, Chichester, UK, pp. 68-151.

Norouzi, H. R., Zarghami, R., SotudehGharebagh, R., Mostoufi, N., sep 2016d. Introduction. In: Coupled CFD-DEM Modeling. John Wiley \& Sons, Ltd, Chichester, UK, pp. 1-13.

O'Rourke, P., 1981. Collective drop effects on vaporizing liquid sprays. Ph.D. thesis, Princeton University.
O'Rourke, P., 1985. The KIVA computer program for multidimensional chemically reactive fluid flows with fuel sprays. In: Glowinski, R., Larrouturou, B., Temam, R. (Eds.), Numerical Simulation of Combustion Phenomena. Vol. 241 of Lecture Notes in Physics. Springer Berlin Heidelberg, pp. 74 89.

Pan, Y., Dudukovic, M. P., Chang, M., 1999. Dynamic simulation of bubbly flow in bubble columns. Chemical Engineering Science 54 (13-14), 24812489.

Parthasarathy, R., Ahmed, N., 1996. Size Distribution of Bubbles Generated by Fine-Pore Spargers. Journal of chemical engineering of Japan 29 (6), 10301034.

Peña-Monferrer, C., Martínez-Cuenca, Rául José Luis, M.-C., Chiva, S., 2016a. Study of bubbly flow through a perforated plate using a Two-Fluid Model and a Discrete Element Method. In: International Conference on Multiphase Flow ICMF2016.

Peña-Monferrer, C., Monrós-Andreu, G., Chiva, S., Martínez-Cuenca, R., José Luis, M.-C., 2017. A CFD-DEM solver to model bubbly flow: Part II. Critical validation in upward vertical pipes including axial evolution. Chemical Engineering Science.

Peña-Monferrer, C., Passalacqua, A., Chiva, S., Muñoz-Cobo, J., 2016b. CFD modelling and validation of upward bubbly flow in an adiabatic vertical pipe using the quadrature method of moments. Nuclear Engineering and Design 301, 320-332.

Peng, Z., Doroodchi, E., Luo, C., Moghtaderi, B., 2014. Influence of void fraction calculation on fidelity of CFD-DEM simulation of gas-solid bubbling fluidized beds. AIChE Journal 60 (6), 2000-2018.

Pfleger, D., Gomes, S., Gilbert, N., Wagner, H.-G., 1999. Hydrodynamic simulations of laboratory scale bubble columns fundamental studies of the Eulerian-Eulerian modelling approach. Chemical Engineering Science 54 (21), 5091-5099.

Pope, S. B., 2000. Turbulent Flows. Cambridge University Press.

Prosperetti, A., Tryggvason, G., 2007. Computational Methods for Multiphase Flow. Cambridge University Press.

Ribeiro Jr., C. P., Lage, P. L. C., 2004. Experimental study on bubble size distributions in a direct-contact evaporator. Brazilian Journal of Chemical Engineering 21, 69-81.

Serizawa, A., Kataoka, I., Michiyoshi, I., 1975. Turbulence structure of airwater bubbly flow. Parts I-III. International Journal of Multiphase Flow 2 (3), $235-246$.

Subramaniam, S., 2013. Lagrangian-Eulerian methods for multiphase flows. Progress in Energy and Combustion Science 39 (2-3), 215-245.

Sugioka, K.-I., Komori, S., jun 2009. Drag and lift forces acting on a spherical gas bubble in homogeneous shear liquid flow. Journal of Fluid Mechanics 629, 173.

Taitel, Y., Bornea, D., Dukler, A. E., 1980. Modelling flow pattern transitions for steady upward gas-liquid flow in vertical tubes. AIChE Journal 26 (3), 345-354.

Thomson, D. J., 1987. Criteria for the selection of stochastic models of particle trajectories in turbulent flows. Journal of Fluid Mechanics 180, 529-556.

Tomiyama, A., Kataoka, I., Zun, I., Sakaguchi, T., 1998. Drag Coefficients of Single Bubbles under Normal and Micro Gravity Conditions. JSME Int. J. Ser. B 41 (2), 472-479.

Tomiyama, A., Tamai, H., Zun, I., Hosokawa, S., 2002. Transverse migration of single bubbles in simple shear flows. Chemical Engineering Science 57 (11), $1849-1858$.

Troshko, A. A., Hassan, Y. A., 2001a. A two-equation turbulence model of turbulent bubbly flows. International Journal of Multiphase Flow 27 (11), 1965-2000.

Troshko, A. A., Hassan, Y. A., 2001b. Law of the wall for two-phase turbulent boundary layers. International Journal of Heat and Mass Transfer 44 (4), 871-875.

Tsuji, Y., Kawaguchi, T., Tanaka, T., 1993. Discrete particle simulation of twodimensional fluidized bed. Powder Technology 77 (1), 79-87.

Uga, T., 1972. Determination of bubble-size distribution in a BWR. Nuclear Engineering and Design 22 (2), 252-261.

Vaidya, A. M., Subbarao, P. M. V., Gaur, R. R., 2006. A Novel and Efficient Method for Particle Locating and Advancing over Deforming, NonOrthogonal Mesh. Numerical Heat Transfer, Part B: Fundamentals 49 (1), 67-88.

van Wijngaarden, L., 1998. On Pseudo Turbulence. Theoretical and Computational Fluid Dynamics 10 (1), 449-458.

Wellek, R. M., Agrawal, A. K., Skelland, A. H. P., sep 1966. Shape of liquid 
drops moving in liquid media. AIChE Journal 12 (5), 854-862.

Williams, F. A., 1958. Spray Combustion and Atomization. Physics of Fluids (1958-1988) 1 (6), 541-545.

Wilson, J. D., Thurtell, G. W., Kidd, G. E., 1981. Numerical simulation of particle trajectories in inhomogeneous turbulence, II: Systems with variable turbulent velocity scale. Boundary-Layer Meteorology 21 (4), 423-441.

Wu, C. L., Berrouk, A. S., Nandakumar, K., 2009a. Three-dimensional discrete particle model for gassolid fluidized beds on unstructured mesh. Chemical Engineering Journal 152 (23), 514-529.

Wu, C. L., Zhan, J. M., Li, Y. S., Lam, K. S., Berrouk, A. S., 2009b. Accurate void fraction calculation for three-dimensional discrete particle model on unstructured mesh. Chemical Engineering Science 64 (6), 1260-1266.

Xiao, H., Sun, J., feb 2011. Algorithms in a Robust Hybrid CFD-DEM Solver for Particle-Laden Flows. Communications in Computational Physics 9 (2), 297-323.

Xu, B., Yu, A., 1997. Numerical simulation of the gas-solid flow in a fluidized bed by combining discrete particle method with computational fluid dynamics. Chemical Engineering Science 52 (16), 2785 - 2809.

Yamoah, S., Martínez-Cuenca, R., Monrós, G., Chiva, S., Macián-Juan, R., jun 2015. Numerical investigation of models for drag, lift, wall lubrication and turbulent dispersion forces for the simulation of gasliquid two-phase flow. Chemical Engineering Research and Design 98, 17-35.

Yang, G., Chen, I.-M., 2006. Equivolumetric partition of solid spheres with applications to orientation workspace analysis of robot manipulators. IEEE Transactions on Robotics 22 (5), 869-879.

Yao, W., Morel, C., 2004. Volumetric interfacial area prediction in upward bubbly two-phase flow. International Journal of Heat and Mass Transfer 47 (2), 307-328.

Zhang, D., Deen, N. G., Kuipers, J. A. M., 2006. Numerical simulation of the dynamic flow behavior in a bubble column: A study of closures for turbulence and interface forces. Chemical Engineering Science 61 (23), 7593 7608 . 

- Development of a CFD-DEM solver to model bubbly flow.

- Implementation of an equivolumetric subelement method.

- Development of a seeding algorithm in pipes and fluid flow influence investigation.

- Modeling of bubble expansion with a simplified model.

- Study of bubble dynamics and lateral forces related with turbulence and bubble size. 